\title{
Minimum Parametrization of the Cauchy Stress Operator
}

\author{
J.-F. Pommaret $\mathbb{1}$ \\ CERMICS, Ecole des Ponts Paris Tech, France \\ Email: jean-francois.pommaret@wanadoo.fr
}

How to cite this paper: Pommaret, J.-F. (2021) Minimum Parametrization of the Cauchy Stress Operator. Journal of Modern Physics, 12, 453-482.

https://doi.org/10.4236/jmp.2021.124032

Received: February 5, 2021

Accepted: March 15, 2021

Published: March 18, 2021

Copyright $\odot 2021$ by author(s) and Scientific Research Publishing Inc. This work is licensed under the Creative Commons Attribution International License (CC BY 4.0).

http://creativecommons.org/licenses/by/4.0/ (c) (i) Open Access

\begin{abstract}
When $\mathcal{D}: \xi \rightarrow \eta$ is a linear differential operator, a "direct problem" is to find the generating compatibility conditions (CC) in the form of an operator $\mathcal{D}_{1}: \eta \rightarrow \zeta$ such that $\mathcal{D} \xi=\eta$ implies $\mathcal{D}_{1} \eta=0$. When $\mathcal{D}$ is involutive, the procedure provides successive first order involutive operators $\mathcal{D}_{1}, \cdots, \mathcal{D}_{n}$ when the ground manifold has dimension $n$, a result first found by M. Janet as early as in 1920, in a footnote. However, the link between this "Janet sequence" and the "Spencer sequence" first found by the author of this paper in 1978 is still not acknowledged. Conversely, when $\mathcal{D}_{1}$ is given, a more difficult "inverse problem" is to look for an operator $\mathcal{D}: \xi \rightarrow \eta$ having the generating CC $\mathcal{D}_{1} \eta=0$. If this is possible, that is when the differential module defined by $\mathcal{D}_{1}$ is torsion-free, one shall say that the operator $\mathcal{D}_{1}$ is parametrized by $\mathcal{D}$ and there is no relation in general between $\mathcal{D}$ and $\mathcal{D}_{2}$. The parametrization is said to be "minimum" if the differential module defined by $\mathcal{D}$ has a vanishing differential rank and is thus a torsion module. The solution of this problem, first found by the author of this paper in 1995, is still not acknowledged. As for the applications of the "differential double duality" theory to standard equations of physics (Cauchy and Maxwell equations can be parametrized while Einstein equations cannot), we do not know other references. When $n=1$ as in control theory, the fact that controllability is a "built in" property of a control system, amounting to the existence of a parametrization and thus not depending on the choice of inputs and outputs, even with variable coefficients, is still not acknowledged by engineers. The parametrization of the Cauchy stress operator in arbitrary dimension $n$ has nevertheless attracted, "separately" and without any general "guiding line", many famous scientists (G.B. Airy in 1863 for $n=2$, J.C. Maxwell in 1863, G. Morera and E. Beltrami in 1892 for $n=3$, A. Einstein in 1915 for $n=4$ ). The aim of this paper is to solve the minimum parametrization problem in arbitrary dimension and to apply it through effective methods that could even
\end{abstract}


be achieved by using computer algebra. Meanwhile, we prove that all these works are using the Einstein operator which is self-adjoint and not the Ricci operator, a fact showing that the Einstein operator, which cannot be parametrized, has already been exhibited by Beltrami more than 20 years before Einstein. As a byproduct, they are all based on the same confusion between the so-called div operator induced from the Bianchi operator $\mathcal{D}_{2}$ and the Cauchy operator which is the formal adjoint of the Killing operator $\mathcal{D}$ parametrizing the Riemann operator $\mathcal{D}_{1}$ for an arbitrary $n$. We prove that this purely mathematical result deeply questions the origin and existence of gravitational waves. We also present the similar motivating situation met in the study of contact structures when $n=3$. Like the Michelson and Morley experiment, it is thus an open historical problem to know whether Einstein was aware of these previous works or not, but the comparison needs no comment.

\section{Keywords}

Differential Operator, Differential Sequence, Killing Operator, Riemann Operator, Bianchi Operator, Cauchy Operator, Electromagnetism, Elasticity, General Relativity, Gravitational Waves

\section{Introduction}

We start recalling the basic tools from the formal theory of systems of partial differential (PD) equations and differential modules needed in order to understand and solve the parametrization problem presented in the abstract. As these new tools are difficult and not so well known, we advise the interested reader to follow them step by step on the explicit motivating examples illustrating this paper, in particular the example of the system of infinitesimal Lie equations defining contact transformations when $n=3$. The main difficulty for the reader not familiar with these new tools is that certain concepts are evident in one framework but not at all in the other and conversely. Considering the single input/single output (SISO) classical control system $\ddot{y}-\dot{u}=0$ with standard notations for ordinary differential (OD) equations, we notice that both $y$ and $u$ can be given arbitrarily separately but that the new quantity $z=\dot{y}-u$ cannot as it must satisfy the autonomous OD equation $\dot{z}=0$ that, of course, cannot be controlled. This is the reason for which a controllable system cannot surely provide such elements called "torsion elements" in module theory. The fact that the controllability just amounts to the lack of any torsion element or, equivalently, to the possibility to parametrize the control system, is left to the reader as a tricky exercise leading to compare with the basic system $\dot{y}-u=0$ which is controllable and can be simply parametrized by the only arbitrary potential $y$ through the formula $\dot{y}=u, y=y$. As we shall see, the surprising fact is that the lack of torsion elements (or the generating ones) can only be tested by the possibility to parametrize the given equations (or to "measure" how it cannot be pa- 
rametrized) and no other classical method can work.

In Section 2, once we shall have found the possibility to parametrize the system or the corresponding operator, that is to say once we know that the corresponding differential module is torsion-free, we shall discover that many possible parametrizations may exist. The idea will then be to modify the formal test in order to compute the minimum number of potentials needed and to find a constructive way to obtain at least one such minimum parametrization.

In Section 3, we shall study with more details the parametrization problems that are existing in continuum mechanics for an arbitrary dimension $n$ of the ground manifold, the case $n=2$ allowing to understand why the Airy operator is just the formal adjoint of the Riemann operator, the case $n=3$ allowing to compare the results respectively obtained by Beltrami, Maxwell and Morera, the case $n=4$ allowing to understand why such a parametrization problem is leading to the self-adjoint Einstein operator and why the so-called "gravitational waves" operator is nothing else than the formal adjoint of the Ricci operator without any reference to Einstein equations.

In Section 4, we shall finally add a few unexpected results coming from the use of the symbol sequences existing for certain generic covectors.

\subsection{System Theory}

If $X$ is a manifold of dimension $n$ with local coordinates $(x)=\left(x^{1}, \cdots, x^{n}\right)$, we denote as usual by $T=T(X)$ the tangent bundle of $X$, by $T^{*}=T^{*}(X)$ the cotangent bundle, by $\wedge^{r} T^{*}$ the bundle of $r$-forms and by $S_{q} T^{*}$ the bundle of $q$-symmetric tensors. More generally, let $E$ be a vector bundle over $X$ with local coordinates $\left(x^{i}, y^{k}\right)$ for $i=1, \cdots, n$ and $k=1, \cdots, m$ simply denoted by $(x, y)$, projection $\pi: E \rightarrow X:(x, y) \rightarrow(x)$ and changes of local coordinate $\bar{x}=\varphi(x), \bar{y}=A(x) y$. We shall denote by $E^{*}$ the vector bundle obtained by inverting the matrix $A$ of the changes of coordinates, exactly like $T^{*}$ is obtained from $T$. We denote by $f: X \rightarrow E:(x) \rightarrow(x, y=f(x))$ a global section of $E$, that is a map such that $\pi \circ f=i d_{X}$ but local sections over an open set $U \subset X$ may also be considered when needed. Under a change of coordinates, a section transforms like $\bar{f}(\varphi(x))=A(x) f(x)$ and the changes of the derivatives can also be obtained with more work. We shall denote by $J_{q}(E)$ the $q$-jet bundle of $E$ with local coordinates $\left(x^{i}, y^{k}, y_{i}^{k}, y_{i j}^{k}, \cdots\right)=\left(x, y_{q}\right)$ called jet coordinates and sections $f_{q}:(x) \rightarrow\left(x, f^{k}(x), f_{i}^{k}(x), f_{i j}^{k}(x), \cdots\right)=\left(x, f_{q}(x)\right)$ transforming like the sections $j_{q}(f):(x) \rightarrow\left(x, f^{k}(x), \partial_{i} f^{k}(x), \partial_{i j} f^{k}(x), \cdots\right)=\left(x, j_{q}(f)(x)\right)$ where both $f_{q}$ and $j_{q}(f)$ are over the section $f$ of $E$. For any $q \geq 0, J_{q}(E)$ is a vector bundle over $X$ with projection $\pi_{q}$ while $J_{q+r}(E)$ is a vector bundle over $J_{q}(E)$ with projection $\pi_{q}^{q+r}, \forall r \geq 0$.

DEFINITION 1.1.1: A linear system of order $q$ on $E$ is a vector sub-bundle $R_{q} \subset J_{q}(E)$ and a solution of $R_{q}$ is a section $f$ of $E$ such that $j_{q}(f)$ is a section of $R_{q}$. With a slight abuse of language, the set of local solutions will be denoted by $\Theta \subset E$. 
Let $\mu=\left(\mu_{1}, \cdots, \mu_{n}\right)$ be a multi-index with length $|\mu|=\mu_{1}+\cdots+\mu_{n}$, class $i$ if $\mu_{1}=\cdots=\mu_{i-1}=0, \mu_{i} \neq 0$ and $\mu+1_{i}=\left(\mu_{1}, \cdots, \mu_{i-1}, \mu_{i}+1, \mu_{i+1}, \cdots, \mu_{n}\right)$. We set $y_{q}=\left\{y_{\mu}^{k}|1 \leq k \leq m, 0 \leq| \mu \mid \leq q\right\}$ with $y_{\mu}^{k}=y^{k}$ when $|\mu|=0$. If $E$ is a vector bundle over $X$ and $J_{q}(E)$ is the $q$-jet bundle of $E$, then both sections $f_{q} \in J_{q}(E)$ and $j_{q}(f) \in J_{q}(E)$ are over the section $f \in E$. There is a natural way to distinguish them by introducing the Spencer operator $d: J_{q+1}(E) \rightarrow T^{*} \otimes J_{q}(E)$ with components $\left(d f_{q+1}\right)_{\mu, i}^{k}(x)=\partial_{i} f_{\mu}^{k}(x)-f_{\mu+1_{i}}^{k}(x)$. The kernel of $d$ consists of sections such that $f_{q+1}=j_{1}\left(f_{q}\right)=j_{2}\left(f_{q-1}\right)=\cdots=j_{q+1}(f)$. Finally, if $R_{q} \subset J_{q}(E)$ is a system of order $q$ on $E$ locally defined by linear equations $\Phi^{\tau}\left(x, y_{q}\right) \equiv a_{k}^{\tau \mu}(x) y_{\mu}^{k}=0$ and local coordinates $(x, z)$ for the parametric jets up to order $q$, the $r$-prolongation $R_{q+r}=\rho_{r}\left(R_{q}\right)=J_{r}\left(R_{q}\right) \cap J_{q+r}(E) \subset J_{r}\left(J_{q}(E)\right)$ is locally defined when $r=1$ by the linear equations $\Phi^{\tau}\left(x, y_{q}\right)=0$,

$d_{i} \Phi^{\tau}\left(x, y_{q+1}\right) \equiv a_{k}^{\tau \mu}(x) y_{\mu+1_{i}}^{k}+\partial_{i} a_{k}^{\tau \mu}(x) y_{\mu}^{k}=0$ and has symbol $g_{q+r}=R_{q+r} \cap S_{q+r} T^{*} \otimes E \subset J_{q+r}(E)$ if one looks at the top order terms. If $f_{q+1} \in R_{q+1}$ is over $f_{q} \in R_{q}$, differentiating the identity $a_{k}^{\tau \mu}(x) f_{\mu}^{k}(x) \equiv 0$ with respect to $x^{i}$ and subtracting the identity $a_{k}^{\tau \mu}(x) f_{\mu+1_{i}}^{k}(x)+\partial_{i} a_{k}^{\tau \mu}(x) f_{\mu}^{k}(x) \equiv 0$, we obtain the identity $a_{k}^{\tau \mu}(x)\left(\partial_{i} f_{\mu}^{k}(x)-f_{\mu+1_{i}}^{k}(x)\right) \equiv 0$ and thus the restriction $d: R_{q+1} \rightarrow T^{*} \otimes R_{q}$. More generally, we have the restriction:

$$
\begin{aligned}
& d: \wedge^{s} T^{*} \otimes R_{q+1} \rightarrow \wedge^{s+1} T^{*} \otimes R_{q}:\left(f_{\mu, I}^{k}(x) d x^{I}\right) \\
& \rightarrow\left(\left(\partial_{i} f_{\mu, I}^{k}(x)-f_{\mu+1_{i}, I}^{k}(x)\right) d x^{i} \wedge d x^{I}\right)
\end{aligned}
$$

using standard multi-index notation for exterior forms, namely $I=\left\{i_{1}<i_{2}<\cdots<i_{r}\right\}$, $d x^{I}=d x^{i_{1}} \wedge \cdots \wedge d x^{i_{r}} \in \wedge^{r} T^{*}$ for a finite basis, and one can easily check that $d \circ d=0$. The restriction of $-d$ to the symbol is called the Spencer map $\delta: \wedge^{s} T^{*} \otimes g_{q+1} \rightarrow \wedge^{s+1} T^{*} \otimes g_{q}$ and $\delta \circ \delta=0$ similarly, leading to the algebraic $\delta$-cohomology $H_{q+r}^{s}\left(g_{q}\right)$ [1]-[7].

DEFINITION 1.1.2: A system $R_{q}$ is said to be formally integrable when all the equations of order $q+r$ are obtained by $r$ prolongations only, $\forall r \geq 0$ or, equivalently, when the projections $\pi_{q+r}^{q+r+s}: R_{q+r+s} \rightarrow R q+r$ are epimorphisms $\forall r, s \geq 0$.

Finding an intrinsic test has been achieved by D.C. Spencer in 1970 [7] along coordinate dependent lines sketched by M. Janet in 1920 [8]. The next procedure providing a Pommaret basis and where one may have to change linearly the independent variables if necessary, is intrinsic even though it must be checked in a particular coordinate system called $\delta$-regular [1] [4] [9].

- Equations of class $n$ : Solve the maximum number $\beta_{q}^{n}$ of equations with respect to the jets of order $q$ and class $n$. Then call $\left(x^{1}, \cdots, x^{n}\right)$ multiplicative variables.

- Equations of class $i \geq 1$ : Solve the maximum number $\beta_{q}^{i}$ of remaining equations with respect to the jets of order $q$ and class $i$. Then call $\left(x^{1}, \cdots, x^{i}\right)$ multiplicative variables and $\left(x^{i+1}, \cdots, x^{n}\right)$ non-multiplicative variables.

- Remaining equations of order $\leq q-1$ : Call $\left(x^{1}, \cdots, x^{n}\right)$ non-multiplicative 
variables.

In actual practice, we shall use a Janet tabular where the multiplicative "variables" are in upper left position while the non-multiplicative variables are represented by dots in lower right position.

DEFINITION 1.1.3: A system of PD equations is said to be involutive if its first prolongation can be obtained by prolonging its equations only with respect to the corresponding multiplicative variables. In that case, we may introduce the characters $\alpha_{q}^{i}=m \frac{(q+n-i-1) !}{(q-1) !(n-i) !}-\beta_{q}^{i} \quad$ for $\quad i=1, \cdots, n \quad$ with $\quad \alpha_{q}^{1} \geq \cdots \geq \alpha_{q}^{n} \geq 0$ and we have $\operatorname{dim}\left(g_{q}\right)=\alpha_{q}^{1}+\cdots+\alpha_{q}^{n}$ while $\operatorname{dim}\left(g_{q+1}\right)=\alpha_{q}^{1}+\cdots+n \alpha_{q}^{n}$.

REMARK 1.1.4: As long as the Prolongation/Projection (PP) procedure has not been achieved in order to get an involutive system, nothing can be said about the CC (fine examples can be found in [6] and the recent [10]). A proof that the second order system defined by Einstein equations is involutive has been given by J. Gasqui in 1982 but this paper cannot be applied to the minimum parametrizations that need specific $\delta$-regular coordinates as we shall see [11].

When $R_{q}$ is involutive, the linear differential operator $\mathcal{D}: E \stackrel{J_{q}}{\rightarrow} J_{q}(E) \stackrel{\Phi}{\rightarrow} J_{q}(E) / R_{q}=F_{0}$ of order $q$ is said to be involutive. Introducing the Janet bundles:

$$
F_{r}=\wedge^{r} T^{*} \otimes J_{q}(E) /\left(\wedge^{r} T^{*} \otimes R_{q}+\delta\left(\wedge^{r-1} T^{*} \otimes S_{q+1} T^{*} \otimes E\right)\right)
$$

we obtain the canonical linear Janet sequence (introduced in [1], p 185 + p 391):

$$
0 \rightarrow \Theta \rightarrow E \stackrel{\mathcal{D}}{\rightarrow} F_{0} \stackrel{\mathcal{D}_{1}}{\rightarrow} F_{1} \stackrel{\mathcal{D}_{2}}{\rightarrow} \cdots \stackrel{\mathcal{D}_{n}}{\rightarrow} F_{n} \rightarrow 0
$$

where each other operator, induced by the Spencer operator, is first order involutive and generates the compatibility conditions (CC) of the preceding one. Similarly, introducing the Spencer bundles:

$$
C_{r}=\wedge^{r} T^{*} \otimes R_{q} / \delta\left(\wedge^{r-1} T^{*} \otimes g_{q+1}\right)
$$

we obtain the canonical linear Spencer sequence also induced by the Spencer operator:

$$
0 \rightarrow \Theta \stackrel{j_{q}}{\rightarrow} C_{0} \stackrel{D_{1}}{\rightarrow} C_{1} \stackrel{D_{2}}{\rightarrow} \cdots \stackrel{D_{n}}{\rightarrow} C_{n} \rightarrow 0
$$

\subsection{Module Theory}

Let $K$ be a differential field with $n$ commuting derivations $\left(\partial_{1}, \cdots, \partial_{n}\right)$ and consider the ring $D=K\left[d_{1}, \cdots, d_{n}\right]=K[d]$ of differential operators with coefficients in $K$ with $n$ commuting formal derivatives satisfying $d_{i} a=a d_{i}+\partial_{i} a$ in the operator sense. If $P=a^{\mu} d_{\mu} \in D=K[d]$, the highest value of $|\mu|$ with $a^{\mu} \neq 0$ is called the order of the operator $P$ and the ring $D$ with multiplication $(P, Q) \rightarrow P \circ Q=P Q$ is filtred by the order $q$ of the operators. We have the filtration $0 \subset K=D_{0} \subset D_{1} \subset \cdots \subset D_{q} \subset \cdots \subset D_{\infty}=D$. As an algebra, $D$ is generated by $K=D_{0}$ and $T=D_{1} / D_{0}$ with $D_{1}=K \oplus T$ if we identify an element $\xi=\xi^{i} d_{i} \in T$ with the vector field $\xi=\xi^{i}(x) \partial_{i}$ of differential geometry, but 
with $\xi^{i} \in K$ now. It follows that $D={ }_{D} D_{D}$ is a bimodule over itself, being at the same time a left $D$-module by the composition $P \rightarrow Q P$ and a right $D$ -module by the composition $P \rightarrow P Q$. We define the adjoint functor ad $: D \rightarrow D^{o p}: P=a^{\mu} d_{\mu} \rightarrow a d(P)=(-1)^{|\mu|} d_{\mu} a^{\mu}$ and we have $\operatorname{ad}(\operatorname{ad}(P))=P$ both with $\operatorname{ad}(P Q)=\operatorname{ad}(Q) \operatorname{ad}(P), \forall P, Q \in D$. Such a definition can be extended to any matrix of operators by using the transposed matrix of adjoint operators (see [5] [9] [12] [13] [14] [15] [16] for more details and applications to control theory or mathematical physics).

Accordingly, if $y=\left(y^{1}, \cdots, y^{m}\right)$ are differential indeterminates, then $D$ acts on $y^{k}$ by setting $d_{i} y^{k}=y_{i}^{k} \rightarrow d_{\mu} y^{k}=y_{\mu}^{k}$ with $d_{i} y_{\mu}^{k}=y_{\mu+1_{i}}^{k}$ and $y_{0}^{k}=y^{k}$. We may therefore use the jet coordinates in a formal way as in the previous section. Therefore, if a system of $\mathrm{OD} / \mathrm{PD}$ equations is written in the form $\Phi^{\tau} \equiv a_{k}^{\tau \mu} y_{\mu}^{k}=0$ with coefficients $a \in K$, we may introduce the free differential module $D y=D y^{1}+\cdots+D y^{m} \simeq D^{m}$ and consider the differential module of equations $I=D \Phi \subset D y$, both with the residual differential module $M=D y / D \Phi$ or $D$-module and we may set $M={ }_{D} M$ if we want to specify the ring of differential operators. We may introduce the formal prolongation with respect to $d_{i}$ by setting $d_{i} \Phi^{\tau} \equiv a_{k}^{\tau \mu} y_{\mu+1_{i}}^{k}+\left(\partial_{i} a_{k}^{\tau \mu}\right) y_{\mu}^{k}$ in order to induce maps $d_{i}: M \rightarrow M: \bar{y}_{\mu}^{k} \rightarrow \bar{y}_{\mu+1_{i}}^{k}$ by residue with respect to $I$ if we use to denote the residue $D y \rightarrow M: y^{k} \rightarrow \bar{y}^{k}$ by a bar like in algebraic geometry. However, for simplicity, we shall not write down the bar when the background will indicate clearly if we are in $D y$ or in $M$. As a byproduct, the differential modules we shall consider will always be finitely generated $(k=1, \cdots, m<\infty)$ and finitely presented $(\tau=1, \cdots, p<\infty)$. Equivalently, introducing the matrix of operators $\mathcal{D}=\left(a_{k}^{\tau \mu} d_{\mu}\right)$ with $m$ columns and $p$ rows, we may introduce the morphism $D^{p} \stackrel{\mathcal{D}}{\rightarrow} D^{m}:\left(P_{\tau}\right) \rightarrow\left(P_{\tau} \Phi^{\tau}\right)$ over $D$ by acting with $D$ on the left of these row vectors while acting with $\mathcal{D}$ on the right of these row vectors by composition of operators with $\operatorname{im}(\mathcal{D})=I$. The presentation of $M$ is defined by the exact cokernel sequence $D^{p} \rightarrow D^{m} \rightarrow M \rightarrow 0$. We notice that the presentation only depends on $K, D$ and $\Phi$ or $\mathcal{D}$, that is to say never refers to the concept of (explicit local or formal) solutions. It follows from its definition that $M$ can be endowed with a quotient filtration obtained from that of $D^{m}$ which is defined by the order of the jet coordinates $y_{q}$ in $D_{q} y$. We have therefore the inductive limit $0 \subseteq M_{0} \subseteq M_{1} \subseteq \cdots \subseteq M_{q} \subseteq \cdots \subseteq M_{\infty}=M$ with $d_{i} M_{q} \subseteq M_{q+1}$ and $M=D M_{q}$ for $q \gg 0$ with prolongations $D_{r} M_{q} \subseteq M_{q+r}, \forall q, r \geq 0$.

DEFINITION 1.2.1: An exact sequence of morphisms finishing at $M$ is said to be a resolution of $M$. If the differential modules involved apart from $M$ are free, that is isomorphic to a certain power of $D$, we shall say that we have a free resolution of $M$.

Having in mind that $K$ is a left $D$-module with the action $(D, K) \rightarrow K:\left(d_{i}, a\right) \rightarrow \partial_{i} a$ and that $D$ is a bimodule over itself, we have only two possible constructions.

DEFINITION 1.2.2: We may define the right (care!) differential module 
$\operatorname{hom}_{D}(M, D)$.

DEFINITION 1.2.3: We define the system $R=h_{K}(M, K)$ and set $R_{q}=\operatorname{hom}_{K}\left(M_{q}, K\right)$ as the system of order $q$. We have the projective limit $R=R_{\infty} \rightarrow \cdots \rightarrow R_{q} \rightarrow \cdots \rightarrow R_{1} \rightarrow R_{0}$. It follows that $f_{q} \in R_{q}: y_{\mu}^{k} \rightarrow f_{\mu}^{k} \in K$ with $a_{k}^{\tau \mu} f_{\mu}^{k}=0$ defines a section at order $q$ and we may set $f_{\infty}=f \in R$ for a section of $R$. For an arbitrary differential field $K$, such a definition has nothing to $d o$ with the concept of a formal power series solution (care).

PROPOSITION 1.2.4: When $M$ is a left $D$-module, then $R$ is also a left $D$ -module.

Proof: As $D$ is generated by $K$ and $T$ as we already said, let us define:

$$
\begin{gathered}
(a f)(m)=a f(m), \quad \forall a \in K, \forall m \in M \\
(\xi f)(m)=\xi f(m)-f(\xi m), \quad \forall \xi=a^{i} d_{i} \in T, \forall m \in M
\end{gathered}
$$

In the operator sense, it is easy to check that $d_{i} a=a d_{i}+\partial_{i} a$ and that $\xi \eta-\eta \xi=[\xi, \eta]$ is the standard bracket of vector fields. We finally get $\left(d_{i} f\right)_{\mu}^{k}=\left(d_{i} f\right)\left(y_{\mu}^{k}\right)=\partial_{i} f_{\mu}^{k}-f_{\mu+1_{i}}^{k}$ and thus recover exactly the Spencer operator of the previous section though this is not evident at all. We also get $\left(d_{i} d_{j} f\right)_{\mu}^{k}=\partial_{i j} f_{\mu}^{k}-\partial_{i} f_{\mu+1_{j}}^{k}-\partial_{j} f_{\mu+1_{i}}^{k}+f_{\mu+1_{i}+1_{j}}^{k} \Rightarrow d_{i} d_{j}=d_{j} d_{i}, \forall i, j=1, \cdots, n \quad$ and thus $d_{i} R_{q+1} \subseteq R_{q} \Rightarrow d_{i} R \subset R$ induces a well defined operator

$R \rightarrow T^{*} \otimes R: f \rightarrow d x^{i} \otimes d_{i} f$. This operator has been first introduced, up to sign, by F.S. Macaulay as early as in 1916 but this is still not acknowledged [17]. For more details on the Spencer operator and its applications, the reader may look at [15] [18] [19] [20] [21].

Q.E.D.

DEFINITION 1.2.5: With any differential module $M$ we shall associate the graded module $G=\operatorname{gr}(M)$ over the polynomial ring $\operatorname{gr}(D) \simeq K[\chi]$ by setting $G=\oplus_{q=0}^{\infty} G_{q}$ with $G_{q}=M_{q} / M_{q-1}$ and we get $g_{q}=G_{q}^{*}$ where the symbol $g_{q}$ is defined by the short exact sequences:

$$
0 \rightarrow M_{q-1} \rightarrow M_{q} \rightarrow G_{q} \rightarrow 0 \Leftrightarrow 0 \rightarrow g_{q} \rightarrow R_{q} \rightarrow R_{q-1} \rightarrow 0
$$

We have the short exact sequences $0 \rightarrow D_{q-1} \rightarrow D_{q} \rightarrow S_{q} T \rightarrow 0$ leading to $g r_{q}(D) \simeq S_{q} T$ and we may set as usual $T^{*}=\operatorname{hom}_{K}(T, K)$ in a coherent way with differential geometry.

The two following definitions, which are well known in commutative algebra, are also valid (with more work) in the case of differential modules (see [5] for more details or the references [9] [22] [23] [24] for an introduction to homological algebra and diagram chasing).

DEFINITION 1.2.6: The set of elements $t(M)=\{m \in M \mid \exists 0 \neq P \in D, P m=0\} \subseteq M$ is a differential module called the torsion submodule of $M$. More generally, a module $M$ is called a torsion module if $t(M)=M$ and a torsion-free module if $t(M)=0$. In the short exact sequence $0 \rightarrow t(M) \rightarrow M \rightarrow M^{\prime} \rightarrow 0$, the module $M^{\prime}$ is torsion-free. Its defining module of equations $I^{\prime}$ is obtained by adding to $I$ a representative basis of $t(M)$ set up to zero and we have thus $I \subseteq I^{\prime}$. 
DEFINITION 1.2.7: A differential module $F$ is said to be free if $F \simeq D^{r}$ for some integer $r>0$ and we shall define $r k_{D}(F)=r$. If $F$ is the biggest free dfferential module contained in $M$, then $M / F$ is a torsion differential module and $\operatorname{hom}_{D}(M / F, D)=0$. In that case, we shall define the differential rank of $M$ to be $r k_{D}(M)=r k_{D}(F)=r$. Accordingly, if $M$ is defined by a linear involutive operator of order $q$, then $r k_{D}(M)=\alpha_{q}^{n}$.

PROPOSITION 1.2.8: If $0 \rightarrow M^{\prime} \rightarrow M \rightarrow M^{\prime \prime} \rightarrow 0$ is a short exact sequence of differential modules and maps or operators, we have $r k_{D}(M)=r k_{D}\left(M^{\prime}\right)+r k_{D}\left(M^{\prime \prime}\right)$.

In the general situation, let us consider the sequence $M^{\prime} \stackrel{f}{\rightarrow} M \stackrel{g}{\rightarrow} M^{\prime \prime}$ of modules which may not be exact and define $B=i m(f) \subseteq Z=\operatorname{ker}(g) \Rightarrow H=Z / B$.

LEMMA 1.2.9: The kernel of the induced epimorphism $\operatorname{coker}(f) \rightarrow \operatorname{coim}(g)$ is isomorphic to $H$.

Proof. It follows from a snake chase in the commutative and exact diagram where $\operatorname{coim}(g) \simeq i m(g)$ :

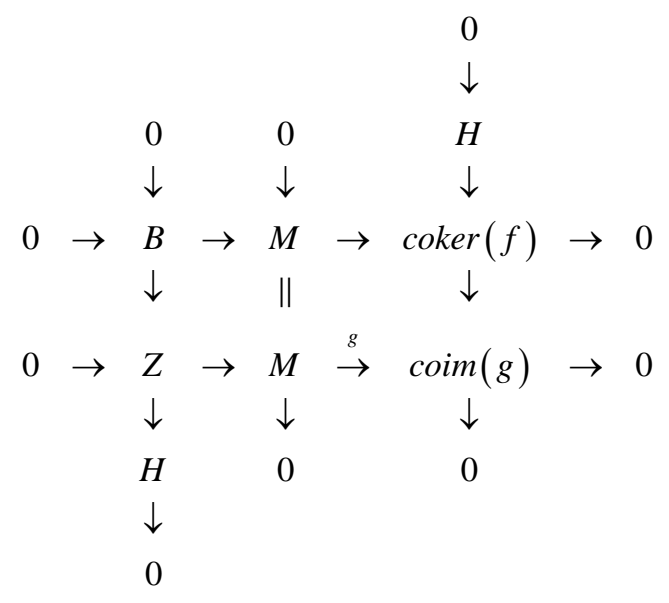

Q.E.D.

In order to conclude this section, we may say that the main difficulty met when passing from the differential framework to the algebraic framework is the "inversion" of arrows. Indeed, when an operator is injective, that is when we have the exact sequence $0 \rightarrow E \stackrel{\mathcal{D}}{\rightarrow} F$ with $\operatorname{dim}(E)=m, \operatorname{dim}(F)=p$, like in the case of the operator $0 \rightarrow E \stackrel{j_{q}}{\rightarrow} J_{q}(E)$, on the contrary, using differential modules, we have the epimorphism $D^{p} \stackrel{\mathcal{D}}{\rightarrow} D^{m} \rightarrow 0$. The case of a formally surjective operator, like the div operator, described by the exact sequence $\stackrel{\mathcal{D}}{\mathrm{D}} \mathrm{F} \rightarrow 0$ is now providing the exact sequence of differential modules

$0 \rightarrow D^{p} \stackrel{\mathcal{D}}{\rightarrow} D^{m} \rightarrow M \rightarrow 0$ because $\mathcal{D}$ has no CC.

\section{Parametrization Problem}

In this section, we shall set up and solve the minimum parametrization problem by comparing the differential geometric approach and the differential algebraic 
approach. In fact, both sides are essential because certain concepts, like "torsion", are simpler in the module approach, as we already said, while others, like "involution" are simpler in the operator approach. However, the reader must never forget that the "extension modules" or the "side changing functor" are pure product of differential homological algebra with no system counterpart. Also, the close link existing between "differential duality" and "adjoint operator" may not be evident at all, even for people quite familiar with mathematical physics [5] [12] [15] [16].

Let us start with a given linear differential operator $\eta \stackrel{\mathcal{D}_{1}}{\rightarrow} \zeta$ between the sections of two given vector bundles $F_{0}$ and $F_{1}$ of respective fiber dimension $m$ and $p$. Multiplying the equations $\mathcal{D}_{1} \eta=\zeta$ by $p$ test functions $\lambda$ considered as a section of the adjoint vector bundle $\operatorname{ad}\left(F_{1}\right)=\wedge^{n} T^{*} \otimes F_{1}^{*}$ and integrating by parts, we may introduce the adjoint vector bundle $\operatorname{ad}\left(F_{0}\right)=\wedge^{n} T^{*} \otimes F_{0}^{*}$ with sections $\mu$ in order to obtain the adjoint operator $\mu \stackrel{a d\left(\mathcal{D}_{1}\right)}{\leftarrow} \lambda$, writing on purpose the arrow backwards, that is from right to left. As any operator is the adjoint of another operator because $\operatorname{ad}(\operatorname{ad}(\mathcal{D}))=\mathcal{D}$, we may decide to denote by $\operatorname{ad}(\mathcal{D})$

$v \stackrel{a d(\mathcal{D})}{\leftarrow} \mu$ the generating CC of $a d\left(\mathcal{D}_{1}\right)$ by introducing a vector bundle $E$ with sections $\xi$ and its adjoint $\operatorname{ad}(E)=\wedge^{n} T^{*} \otimes E^{*}$ with sections $v$. We have thus obtained the formally exact differential sequence:

$$
v \stackrel{a d(\mathcal{D})}{\leftarrow} \mu \stackrel{a d\left(\mathcal{D}_{1}\right)}{\leftarrow} \lambda
$$

and its formally exact adjoint sequence:

$$
\xi \stackrel{\mathcal{D}}{\rightarrow} \eta \stackrel{\mathcal{D}_{1}}{\rightarrow} \zeta
$$

providing a parametrization if and only if $\mathcal{D}_{1}$ generates the CC of $\mathcal{D}_{1}$. Such a situation may not be satisfied but we shall assume it from now on because otherwise $\mathcal{D}_{1}$ cannot be parametrized according to the double differential duality test, for example in the case of the Einstein equations [25] [26] [27] or the extension to the conformal group and other Lie groups of transformations [15] [19] [20] [21] [28] [29] [30]. Nevertheless, for the interested reader only, we provide the following key result on which this procedure is based (see [5] [12] [13] [14] [16] for more details):

THEOREM 2.1: If $M$ is a differential module, we have the exact sequence of differential modules:

$$
0 \rightarrow t(M) \rightarrow M \stackrel{\varepsilon}{\rightarrow} \operatorname{hom}_{D}\left(\operatorname{hom}_{D}(M, D), D\right)
$$

where the map $\varepsilon$ is defined by $\varepsilon(m)(f)=f(m), \forall m \in M, f \in \operatorname{hom}_{D}(M, D)$. Moreover, if $N$ is the differential module defined by $\operatorname{ad}(\mathcal{D})$, then $t(M)=\operatorname{ext}_{D}^{1}(N, D)$.

In order to pass to the differential module framework, let us introduce the free differential modules $D \xi \simeq D^{l}, D \eta \simeq D^{m}, D \zeta \simeq D^{p}$. We have similarly the ad- 
joint free differential modules $D v \simeq D^{l}, D \mu \simeq D^{m}, D \lambda \simeq D^{p}$, because $\operatorname{dim}(\operatorname{ad}(E))=\operatorname{dim}(E)$ and $\operatorname{hom}_{D}\left(D^{m}, D\right) \simeq D^{m}$. Of course, in actual practice, the geometric meaning is totally different because we have volume forms in the dual framework. We have thus obtained the formally exact sequence of differential modules:

$$
D^{p} \stackrel{\mathcal{D}_{1}}{\rightarrow} D^{m} \stackrel{\mathcal{D}}{\rightarrow} D^{l}
$$

and the formally exact adjoint sequence:

$$
D^{p} \stackrel{\operatorname{ad}\left(\mathcal{D}_{1}\right)}{\leftarrow} D^{m} \stackrel{a d(\mathcal{D})}{\leftarrow} D^{l}
$$

The procedure with 4 steps is as follows in the operator language:

- STEP 1: Start with the formally exact parametrizing sequence already constructed by differential biduality. We have thus $\operatorname{im}(\mathcal{D})=\operatorname{ker}\left(\mathcal{D}_{1}\right)$ and the corresponding differential module $M_{1}$ defined by $\mathcal{D}_{1}$ is torsion-free by assumption.

- STEP 2: Construct the adjoint sequence which is also formally exact by assumption.

- STEP 3: Find a maximum set of differentially independent CC $\operatorname{ad}\left(\mathcal{D}^{\prime}\right): \mu \rightarrow v^{\prime}$ among the generating CC $\operatorname{ad}(\mathcal{D}): \mu \rightarrow v$ of $\operatorname{ad}\left(\mathcal{D}_{1}\right)$ in such a way that $\operatorname{im}\left(\operatorname{ad}\left(\mathcal{D}^{\prime}\right)\right)$ is a maximum free differential submodule of $\operatorname{im}(\operatorname{ad}(\mathcal{D}))$ that is any element in $\operatorname{im}(\operatorname{ad}(\mathcal{D}))$ is differentially algebraic over $\operatorname{im}\left(\operatorname{ad}\left(\mathcal{D}^{\prime}\right)\right)$.

- STEP 4: Using differential duality, construct $\mathcal{D}^{\prime}=\operatorname{ad}\left(\operatorname{ad}\left(\mathcal{D}^{\prime}\right)\right)$.

It remains to prove that $\mathcal{D}_{1}$ generates the CC of $\mathcal{D}^{\prime}$ in the following diagram:

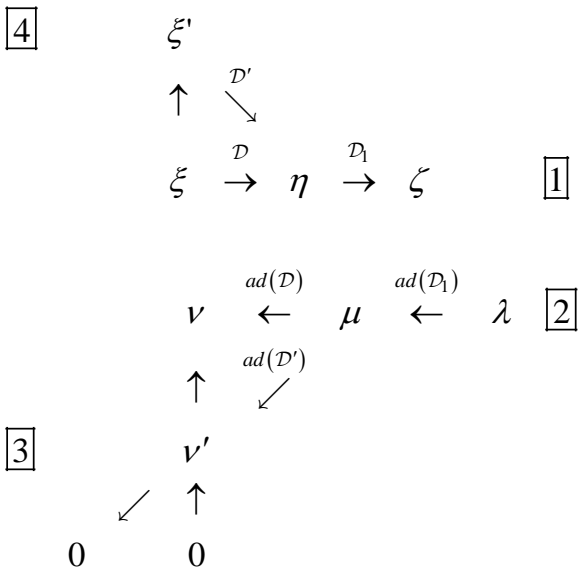

PROPOSITION 2.2: $\mathcal{D}^{\prime}$ is a minimum parametrization of $\mathcal{D}_{1}$.

Proof. Let us denote the number of potentials $\xi$ by $l$ (respectively $\xi^{\prime}$ by $l^{\prime}$ ), the number of unknowns $\eta$ by $m$ and the number of given equations $\zeta$ by $p$. As $\operatorname{ad}\left(\mathcal{D}^{\prime}\right)$ has no CC by construction, then $\operatorname{ad}\left(\mathcal{D}^{\prime}\right): \mu \rightarrow v^{\prime}$ is a formally surjective operator. On the differential module level, we have the injective operator $\operatorname{ad}\left(\mathcal{D}^{\prime}\right): D^{l^{\prime}} \rightarrow D^{m}$ because there are no CC. Applying $\operatorname{hom}_{D}(\bullet, D)$ or duality, we get an operator $D^{m} \rightarrow D^{l^{\prime}}$ with a cokernel which is a torsion module because it has rank $l^{\prime}-r k_{D}\left(\mathcal{D}^{\prime}\right)=l^{\prime}-r k_{D}\left(\operatorname{ad}\left(\mathcal{D}^{\prime}\right)\right)=l^{\prime}-l^{\prime}=0$. 
However, in actual practice as will be seen in the contact case, things are not so simple and we shall use the following commutative and exact diagram of differential modules based on a long ker/coker long exact sequence (compare to [31] and [32]):

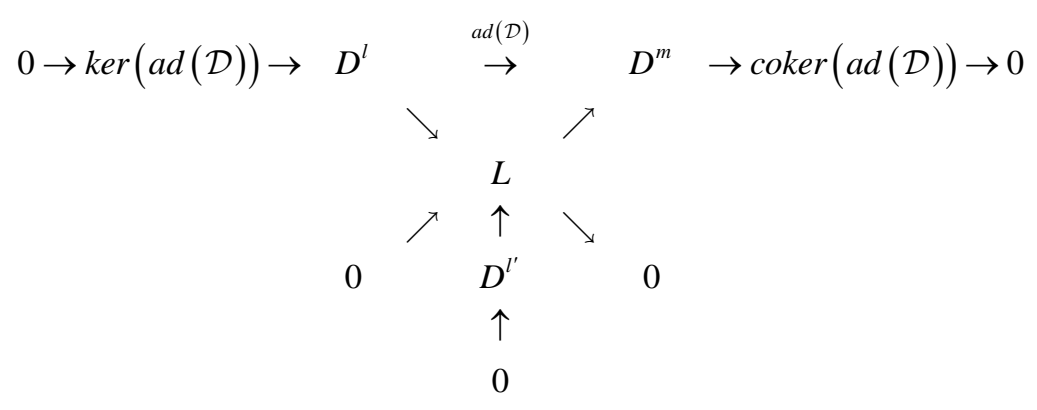

Setting $L=D^{l} / \operatorname{ker}(\operatorname{ad}(\mathcal{D}))$ and introducing the biggest free differential module $D^{l^{\prime}} \subseteq L$ we have $r k_{D}\left(D^{l^{\prime}}\right)=r k_{D}(L) \leq r k_{D}\left(D^{l}\right) \Rightarrow l^{\prime} \leq l$, we may define the injective (care) operator ad $\left(\mathcal{D}^{\prime}\right)$ by the composition of monomorphisms $D^{l^{\prime}} \rightarrow L \rightarrow D^{m}$ where the second is obtained by picking a basis of $D^{l^{\prime}}$, lifting it to $D^{l}$ and pushing it to $D^{m}$ by applying $\operatorname{ad}(\mathcal{D})$. We notice that $L$ can be viewed as the differential module defined by the generating CC of $\operatorname{ad}(\mathcal{D})$ that could also be used as in [31].

Then we have $\operatorname{ad}\left(\mathcal{D}^{\prime}\right) \circ \operatorname{ad}\left(\mathcal{D}_{1}\right)=\operatorname{ad}\left(\mathcal{D}_{1} \circ \mathcal{D}^{\prime}\right)=0 \Rightarrow \mathcal{D}_{1} \circ \mathcal{D}^{\prime}=0$ and thus $\mathcal{D}_{1}$ is surely among the CC of $\mathcal{D}^{\prime}$. Therefore, the differential sequence $\xi^{\prime} \rightarrow \eta \stackrel{\mathcal{D}_{1}}{\rightarrow} \zeta$ on the operator level or the sequence $D^{p} \stackrel{\mathcal{D}_{1}}{\rightarrow} D^{m} \stackrel{\mathcal{D}^{\prime}}{\rightarrow} D^{l^{\prime}}$ on the differential module level may not be exact and we can thus apply the previous Lemma. Changing slightly the notations, we have now

$B=\operatorname{im}\left(\mathcal{D}_{1}\right)=\operatorname{ker}(\mathcal{D}) \subseteq \operatorname{ker}\left(\mathcal{D}^{\prime}\right)=Z$. But we have also $r k_{D}(B)=m-r k_{D}(\mathcal{D})$, $r k(Z)=m-r k_{D}\left(\mathcal{D}^{\prime}\right) \Rightarrow r k_{D}(H)=r k_{D}(\mathcal{D})-r k_{D}\left(\mathcal{D}^{\prime}\right)=0$ by construction.

Taking into account the previous Lemma, we may set $\operatorname{coim}\left(\mathcal{D}_{1}\right)=M_{1} \subseteq D^{l}$ by assumption and consider $\operatorname{im}\left(\mathcal{D}^{\prime}\right)=M_{1}^{\prime} \subseteq D^{l^{\prime}}$ in order to obtain the short exact sequence of differential modules $0 \rightarrow H \rightarrow M_{1} \rightarrow M_{1}^{\prime} \rightarrow 0$. As $H$ is a torsion module and the differential module $M_{1}$ defined by $\mathcal{D}_{1}$ is torsion-free by assumption, the only possibility is that $H=0$ and thus $\operatorname{im}\left(\mathcal{D}_{1}\right)=\operatorname{ker}\left(\mathcal{D}^{\prime}\right)$, that is $\mathcal{D}^{\prime}$ is a minimum parametrization of $\mathcal{D}_{1}$ with $l^{\prime} \leq l$ potentials.

Q.E.D.

\section{EXAMPLE 2.3: Contact transformations}

With $m=n=3, K=\mathbb{Q}\left(x^{1}, x^{2}, x^{3}\right)=\mathbb{Q}(x)$, we may introduce the so-called contact 1 -form $\alpha=d x^{1}-x^{3} d x^{2}$. The system of infinitesimal Lie equations defining the infinitesimal contact transformations is obtained by eliminating the factor $\rho(x)$ in the equations $\mathcal{L}(\xi) \alpha=\rho \alpha$ where $\mathcal{L}$ is the standard Lie derivative. This system is thus only generated by $\eta^{1}$ and $\eta^{2}$ below but is not involutive and one has to introduce $\eta^{3}$ defined by the first order CC:

$$
\zeta \equiv \partial_{3} \eta^{1}-\partial_{2} \eta^{2}-x^{3} \partial_{1} \eta^{2}+\eta^{3}=0
$$

in order to obtain the following involutive system with two equations of class 3 
and one equation of class 2 , a result leading to $\beta_{1}^{3}=2, \beta_{1}^{2}=1, \beta_{1}^{1}=0$ :

$$
\left\{\begin{array}{l}
\eta^{3} \equiv \partial_{3} \xi^{3}+\partial_{2} \xi^{2}+2 x^{3} \partial_{1} \xi^{2}-\partial_{1} \xi^{1}=0 \\
\eta^{2} \equiv \partial_{3} \xi^{1}-x^{3} \partial_{3} \xi^{2}=0 \\
\eta^{1} \equiv \partial_{2} \xi^{1}-x^{3} \partial_{2} \xi^{2}+x^{3} \partial_{1} \xi^{1}-\left(x^{3}\right)^{2} \partial_{1} \xi^{2}-\xi^{3}=0
\end{array}\right.
$$

The characters are thus $\alpha_{1}^{3}=3-2=1<\alpha_{1}^{2}=3-1=2, \alpha_{1}^{1}=3-0=3$ with sum equal to $1+2+3=6=\operatorname{dim}\left(g_{1}\right)=3 \times 3-3$. In this situation, if $M$ is the differential module defined by this system or the corresponding operator $\mathcal{D}$, we know that $r k_{D}(M)=\alpha_{1}^{3}=1=3-2=r k_{D}(D \xi)-r k_{D}(\mathcal{D})$. Of course, a differential transcendence basis for $\mathcal{D}$ can be the operator $\mathcal{D}^{\prime}: \xi \rightarrow\left\{\eta^{2}, \eta^{3}\right\}$ but, in view of the CC, we may equally choose any couple among $\left\{\eta^{1}, \eta^{2}, \eta^{3}\right\}$ and we obtain $r k_{D}\left(\mathcal{D}^{\prime}\right)=r k_{D}(\mathcal{D})=2$ in any case, but now $\mathcal{D}^{\prime}$ is formally surjective, contrary to $\mathcal{D}$. The same result can also be obtained directly from the unique CC or the corresponding operator $\mathcal{D}_{1}$ defining the differential module $M_{1}$. Finally, we have $r k_{D}\left(M_{1}\right)=3-1=2=r k_{D}(D \eta)-r k_{D}\left(\mathcal{D}_{1}\right)$ and we check that we have indeed $r k_{D}(M)+r k_{D}\left(M_{1}\right)=1+2=3=r k_{D}(D \xi)$.

It is well known that such a system can be parametrized by the injective parametrization (see [2] and [3] for more details and the study of the general dimension $n=2 p+1)$ :

$$
-x^{3} \partial_{3} \phi+\phi=\xi^{1},-\partial_{3} \phi=\xi^{2}, \partial_{2} \phi+x^{3} \partial_{1} \phi=\xi^{3} \Rightarrow \xi^{1}-x^{3} \xi^{2}=\phi
$$

It is however not so well known and quite striking that such a parametrization can be recovered independently by using the parametrization of the differential module defined by $\eta^{1}=0$ with potentials $\xi^{1}$ and $\xi^{2}$ while setting:

$$
\left(\xi^{1}, \xi^{2}\right) \rightarrow \xi^{3}=\partial_{2} \xi^{1}-x^{3} \partial_{2} \xi^{2}+x^{3} \partial_{1} \xi^{1}-\left(x^{3}\right)^{2} \partial_{1} \xi^{2}
$$

Taking into account the differential constraint $\eta^{2} \equiv \partial_{3} \xi^{1}-x^{3} \partial_{3} \xi^{2}=0$, that is $\xi^{2}=-\partial_{3}\left(\xi^{1}-x^{3} \xi^{2}\right)$ and substituting in $\eta^{3}=0$, we get no additional constraint. We finally only need to modify the potentials while "defining" now $\phi=\xi^{1}-x^{3} \xi^{2}=\bar{\xi}^{1}$ as before.

The associated differential sequence is:

$$
\begin{aligned}
& 0 \rightarrow \phi \stackrel{\mathcal{D}_{-1}}{\rightarrow} \xi \stackrel{\mathcal{D}}{\rightarrow} \eta \stackrel{\mathcal{D}_{1}}{\rightarrow} \zeta \rightarrow 0 \\
& 0 \rightarrow 1 \rightarrow 3 \rightarrow 3 \rightarrow 1 \rightarrow 0
\end{aligned}
$$

with Euler-Poincaré characteristic $1-3+3-1=0$ but is not a Janet sequence because $\mathcal{D}_{-1}$ is not involutive, its completion to involution being the trivially involutive operator $j_{1}: \phi \rightarrow j_{1}(\phi)$.

Introducing the ring $D=K\left[d_{1}, d_{2}, d_{3}\right]=K[d]$ of linear differential operators with coefficients in the differential field $K$, the corresponding differential module $M \simeq D$ is projective and even free, thus torsion-free or 0-pure, being defined by the split exact sequence of free differential modules:

$$
0 \rightarrow D \stackrel{\mathcal{D}_{1}}{\rightarrow} D^{3} \stackrel{\mathcal{D}}{\rightarrow} D^{3} \stackrel{\mathcal{D}_{-1}}{\rightarrow} D \rightarrow 0
$$

We let the reader prove as an exercise that the adjoint sequence: 


$$
\begin{gathered}
0 \leftarrow \theta \stackrel{a d\left(\mathcal{D}_{-1}\right) \quad a d(\mathcal{D}) \quad{ }^{a d}\left(\mathcal{D}_{1}\right)}{\leftarrow} v \leftarrow \mu \leftarrow 0 \\
0 \leftarrow 1 \leftarrow 3 \leftarrow 3 \leftarrow 1 \leftarrow 0
\end{gathered}
$$

starting from the Lagrange multiplier $\lambda$ is also a split exact sequence of free differential modules.

We finally prove that the situation met for the contact structure is exactly the same as the one that we shall meet in the metric structure, namely that one can identify $\mathcal{D}_{-1}$ not with $\mathcal{D}_{1}$ of course but with $\operatorname{ad}\left(\mathcal{D}_{1}\right)$. For this, let us modify the "basis" linearly by setting $\left(\bar{\xi}^{1}=\xi^{1}-x^{3} \xi^{2}, \bar{\xi}^{2}=\xi^{2}, \bar{\xi}^{3}=\xi^{3}\right)$ and suppressing the bar for simplicity, we obtain the new injective parametrization:

$$
\phi=\xi^{1},-\partial_{3} \phi=\xi^{2}, \partial_{2} \phi+x^{3} \partial_{1} \phi=\xi^{3}
$$

and may eliminate $\phi$ in order to consider the new involutive system, renumbering the equations through a cyclic permutation of $(1,2,3)$ :

$$
\left\{\begin{array}{l}
\eta^{1} \equiv \partial_{3} \xi^{3}+\partial_{2} \xi^{2}+x^{3} \partial_{1} \xi^{2}-\partial_{1} \xi^{1}=0 \\
\eta^{3} \equiv \partial_{3} \xi^{1}+\xi^{2}=0 \\
\eta^{2} \equiv \partial_{2} \xi^{1}+x^{3} \partial_{1} \xi^{1}-\xi^{3}=0
\end{array} \mid \begin{array}{lll}
1 & 2 & 3 \\
1 & 2 & 3 \\
1 & 2 & \bullet
\end{array}\right.
$$

with the unique first order CC defining $\mathcal{D}_{1}$ :

$$
\zeta \equiv \partial_{3} \eta^{2}-\partial_{2} \eta^{3}-x^{3} \partial_{1} \eta^{3}+\eta^{1}=0
$$

Multiplying by $\lambda$ and integrating by parts, we obtain for $\operatorname{ad}\left(\mathcal{D}_{1}\right)$ :

$$
\eta^{1} \rightarrow \lambda=\mu^{1}, \eta^{2} \rightarrow-\partial_{3} \lambda=\mu^{2}, \eta^{3} \rightarrow \partial_{2} \lambda+x^{3} \partial_{1} \lambda=\mu^{3}
$$

obtaining therefore $\mathcal{D}_{-1}=\operatorname{ad}\left(\mathcal{D}_{1}\right) \Leftrightarrow \mathcal{D}_{1}=\operatorname{ad}\left(\mathcal{D}_{-1}\right)$ exactly.

As for $\mathcal{D} \xi=\eta$, we obtain the formal operator matrix:

$$
\left(\begin{array}{ccc}
-d_{1} & d_{2}+x^{3} d_{1} & d_{3} \\
d_{2}+x^{3} d_{1} & 0 & -1 \\
d_{3} & 1 & 0
\end{array}\right)
$$

Similarly, for $\operatorname{ad}(\mathcal{D})$ we obtain the formal operator matrix:

$$
\left(\begin{array}{ccc}
d_{1} & -\left(d_{2}+x^{3} d_{1}\right) & -d_{3} \\
-\left(d_{2}+x^{3} d_{1}\right) & 0 & 1 \\
-d_{3} & -1 & 0
\end{array}\right)
$$

and finally discover that $\operatorname{ad}(\mathcal{D})=-\mathcal{D}$, a striking result showing that both operators have the same CC and parametrization even though $\mathcal{D}$ is not self-adjoint.

\section{Einstein Equations}

Linearizing the Ricci tensor $\rho_{i j}$ over the Minkowski metric $\omega$, we obtain the usual second order homogeneous Ricci operator $\Omega \rightarrow R$ with 4 terms:

$$
\begin{gathered}
2 R_{i j}=\omega^{r s}\left(d_{r s} \Omega_{i j}+d_{i j} \Omega_{r s}-d_{r i} \Omega_{s j}-d_{s j} \Omega_{r i}\right)=2 R_{j i} \\
\operatorname{tr}(R)=\omega^{i j} R_{i j}=\omega^{i j} d_{i j} \operatorname{tr}(\Omega)-\omega^{r u} \omega^{s v} d_{r s} \Omega_{u v}
\end{gathered}
$$


We may define the Einstein operator by setting $E_{i j}=R_{i j}-\frac{1}{2} \omega_{i j} \operatorname{tr}(R)$ and obtain the 6 terms [33]:

$$
\begin{aligned}
2 E_{i j}= & \omega^{r s}\left(d_{r s} \Omega_{i j}+d_{i j} \Omega_{r s}-d_{r i} \Omega_{s j}-d_{s j} \Omega_{r i}\right) \\
& -\omega_{i j}\left(\omega^{r s} \omega^{u v} d_{r s} \Omega_{u v}-\omega^{r u} \omega^{s v} d_{r s} \Omega_{u v}\right)
\end{aligned}
$$

We have the (locally exact) differential sequence of operators acting on sections of vector bundles where the order of an operator is written under its arrow:

$$
\begin{gathered}
T \underset{1}{T} \stackrel{\text { Killing }}{\rightarrow} S_{2} T^{*} \underset{2}{\stackrel{\text { Riemann }}{\rightarrow}} F_{1} \underset{1}{\stackrel{\text { Bianchi }}{\rightarrow}} F_{2} \\
\stackrel{\mathcal{D}}{\rightarrow} n(n+1) / 2 \stackrel{\mathcal{D}_{1}}{\rightarrow} n^{2}\left(n^{2}-1\right) / 12 \stackrel{\mathcal{D}_{2}}{\rightarrow} n^{2}\left(n^{2}-1\right)(n-2) / 24
\end{gathered}
$$

Our purpose is now to study the differential sequence onto which its right part is projecting:

$$
\begin{gathered}
S_{2} T^{*} \underset{2}{\stackrel{\text { Einstein }}{\rightarrow}} S_{2} T^{*} \underset{1}{\stackrel{\text { div }}{\rightarrow}} T^{*} \rightarrow 0 \\
n(n+1) / 2 \rightarrow n(n+1) / 2 \rightarrow n \rightarrow 0
\end{gathered}
$$

and the following adjoint sequence where we have set [15] [19] [20] [31] [34]:

$$
\begin{gathered}
\text { Cauchy }=\text { ad (Killing), Beltrami }=\text { ad }(\text { Riemann }), \text { Lanczos }=\text { ad (Bianchi) } \\
\operatorname{ad}(T) \stackrel{\text { Cauchy }}{\leftarrow} \operatorname{ad}\left(S_{2} T^{*}\right) \stackrel{\text { Beltrami }}{\leftarrow} \operatorname{ad}\left(F_{1}\right) \stackrel{\text { Lanczos }}{\leftarrow} \operatorname{ad}\left(F_{2}\right)
\end{gathered}
$$

In this sequence, if $E$ is a vector bundle over the ground manifold $X$ with dimension $n$, we may introduce the new vector bundle $\operatorname{ad}(E)=\wedge^{n} T^{*} \otimes E^{*}$ where $E^{*}$ is obtained from $E$ by inverting the transition rules exactly like $T^{*}$ is obtained from $T$. We have for example $\operatorname{ad}(T)=\wedge^{n} T^{*} \otimes T^{*} \simeq \wedge^{n} T^{*} \otimes T \simeq \wedge^{n-1} T^{*}$ because $T^{*}$ is isomorphic to $T$ by using the metric $\omega$. The $10 \times 10$ Einstein operator matrix is induced from the $10 \times 20$ Riemann operator matrix and the $10 \times 4$ div operator matrix is induced from the $20 \times 20$ Bianchi operator matrix. We advise the reader not familiar with the formal theory of systems or operators to follow the computation in dimension $n=2$ with the $1 \times 3$ Airy operator matrix, which is the formal adjoint of the $3 \times 1$ Riemann operator matrix, and $n=3$ with the $6 \times 6$ Beltrami operator matrix which is the formal adjoint of the $6 \times 6$ Riemann operator matrix which is easily seen to be self-adjoint up to a change of basis.

With more details, we have:

- $n=2$ : The stress equations become $d_{1} \sigma^{11}+d_{2} \sigma^{12}=0, d_{1} \sigma^{21}+d_{2} \sigma^{22}=0$. Their second order parametrization $\sigma^{11}=d_{22} \phi, \sigma^{12}=\sigma^{21}=-d_{12} \phi, \sigma^{22}=d_{11} \phi$ has been provided by George Biddell Airy in 1863 [35] and is well known [5]. We get the second order system:

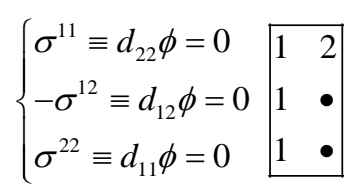


which is involutive with one equation of class 2, 2 equations of class 1 and it is easy to check that the 2 corresponding first order CC are just the Cauchy equations. Of course, the Airy function (1 term) has absolutely nothing to do with the perturbation of the metric ( 3 terms). With more details, when $\omega$ is the Euclidean metric, we may consider the only component:

$$
\begin{aligned}
\operatorname{tr}(R) & =\left(d_{11}+d_{22}\right)\left(\Omega_{11}+\Omega_{22}\right)-\left(d_{11} \Omega_{11}+2 d_{12} \Omega_{12}+d_{22} \Omega_{22}\right) \\
& =d_{22} \Omega_{11}+d_{11} \Omega_{22}-2 d_{12} \Omega_{12}
\end{aligned}
$$

Multiplying by the Airy function $\phi$ and integrating by parts, we discover that:

$$
\text { Airy }=\text { ad }(\text { Riemann }) \Leftrightarrow \text { Riemann }=\text { ad }(\text { Airy })
$$

in the following differential sequences:

$$
\begin{gathered}
2 \underset{1}{\stackrel{\text { Killing }}{\rightarrow}} 3 \underset{2}{\stackrel{\text { Riemann }}{\rightarrow}} 1 \rightarrow 0 \\
0 \leftarrow 2 \underset{1}{\stackrel{\text { Cauchy }}{\leftarrow}} 3 \underset{2}{\stackrel{\text { Airy }}{\leftarrow}} 1
\end{gathered}
$$

- $n=3$ : It is more delicate to parametrize the 3 PD equations:

$$
\begin{aligned}
& d_{1} \sigma^{11}+d_{2} \sigma^{12}+d_{3} \sigma^{13}=0 \\
& d_{1} \sigma^{21}+d_{2} \sigma^{22}+d_{3} \sigma^{23}=0 \\
& d_{1} \sigma^{31}+d_{2} \sigma^{32}+d_{3} \sigma^{33}=0
\end{aligned}
$$

A direct computational approach has been provided by Eugenio Beltrami in 1892 [36] [37], James Clerk Maxwell in 1870 [38] and Giacinto Morera in 1892 [37] [39] by introducing the 6 stress functions $\phi_{i j}=\phi_{j i}$ in the Beltrami parametrization. The corresponding system:

$$
\left\{\begin{array}{l|l|l}
\sigma^{11} \equiv d_{33} \phi_{22}+d_{22} \phi_{33}-2 d_{23} \phi_{23}=0 \\
-\sigma^{12} \equiv d_{33} \phi_{12}+d_{12} \phi_{33}-d_{13} \phi_{23}-d_{23} \phi_{13}=0 \\
\sigma^{22} \equiv d_{33} \phi_{11}+d_{11} \phi_{33}-2 d_{13} \phi_{13}=0 \\
\sigma^{13} \equiv d_{23} \phi_{12}+d_{12} \phi_{23}-d_{22} \phi_{13}-d_{13} \phi_{22}=0 \\
-\sigma^{23} \equiv d_{23} \phi_{11}+d_{11} \phi_{23}-d_{12} \phi_{13}-d_{13} \phi_{12}=0 \\
\sigma^{33} \equiv d_{22} \phi_{11}+d_{11} \phi_{22}-2 d_{12} \phi_{12}=0 \\
1 & 2 & 3 \\
1 & 2 & \bullet \\
1 & 2 & \bullet
\end{array}\right.
$$

is involutive with 3 equations of class 3, 3 equations of class 2 and no equation of class 1 . The three characters are thus $\alpha_{2}^{3}=1 \times 6-3=3<\alpha_{2}^{2}=2 \times 6-3=9<\alpha_{2}^{1}=3 \times 6-0=18$ and we have $\begin{aligned} \operatorname{dim}\left(g_{2}\right) & =\alpha_{2}^{1}+\alpha_{2}^{2}+\alpha_{2}^{3}=18+9+3=30 \\ & =\operatorname{dim}\left(S_{2} T^{*} \otimes S_{2} T^{*}\right)-\operatorname{dim}\left(S_{2} T^{*}\right)=6 \times 6-6 \quad \text { [1]. The } 3 \mathrm{CC} \text { are describing }\end{aligned}$ the stress equations which admit therefore a parametrization... but without any geometric framework, in particular without any possibility to imagine that the above second order operator is nothing else but the formal adjoint of the Riemann operator, namely the (linearized) Riemann tensor with $n^{2}\left(n^{2}-1\right) / 2=6$ independent components when $n=3$ [31]. Breaking the canonical form of the six equations which is associated with the Janet tabular, we may rewrite the Bel- 
trami parametrization of the Cauchy stress equations as follows, after exchanging the third row with the fourth row, keeping the ordering

$\{(11)<(12)<(13)<(22)<(23)<(33)\}$ :

$$
\left(\begin{array}{cccccc}
d_{1} & d_{2} & d_{3} & 0 & 0 & 0 \\
0 & d_{1} & 0 & d_{2} & d_{3} & 0 \\
0 & 0 & d_{1} & 0 & d_{2} & d_{3}
\end{array}\right)\left(\begin{array}{cccccc}
0 & 0 & 0 & d_{33} & -2 d_{23} & d_{22} \\
0 & -d_{33} & d_{23} & 0 & d_{13} & -d_{12} \\
0 & d_{23} & -d_{22} & -d_{13} & d_{12} & 0 \\
d_{33} & 0 & -2 d_{13} & 0 & 0 & d_{11} \\
-d_{23} & d_{13} & d_{12} & 0 & -d_{11} & 0 \\
d_{22} & -2 d_{12} & 0 & d_{11} & 0 & 0
\end{array}\right) \equiv 0
$$

as an identity where 0 on the right denotes the zero operator. However, if $\Omega$ is a perturbation of the metric $\omega$, the standard implicit summation used in continuum mechanics is, when $n=3$ :

$$
\begin{aligned}
\sigma^{i j} \Omega_{i j}= & \sigma^{11} \Omega_{11}+2 \sigma^{12} \Omega_{12}+2 \sigma^{13} \Omega_{13}+\sigma^{22} \Omega_{22}+2 \sigma^{23} \Omega_{23}+\sigma^{33} \Omega_{33} \\
= & \Omega_{22} d_{33} \phi_{11}+\Omega_{33} d_{22} \phi_{11}-2 \Omega_{23} d_{23} \phi_{11}+\cdots \\
& +\Omega_{23} d_{13} \phi_{12}+\Omega_{13} d_{23} \phi_{12}-\Omega_{12} d_{33} \phi_{12}-\Omega_{33} d_{12} \phi_{12}+\cdots
\end{aligned}
$$

because the stress tensor density $\sigma$ is supposed to be symmetric. Integrating by parts in order to construct the adjoint operator, we get:

$$
\begin{aligned}
& \phi_{11} \rightarrow d_{33} \Omega_{22}+d_{22} \Omega_{33}-2 d_{23} \Omega_{23} \\
& \phi_{12} \rightarrow d_{13} \Omega_{23}+d_{23} \Omega_{13}-d_{33} \Omega_{12}-d_{12} \Omega_{33}
\end{aligned}
$$

and so on, obtaining therefore the striking identification:

$$
\text { Riemann }=\operatorname{ad}(\text { Beltrami }) \Leftrightarrow \text { Beltrami }=\text { ad }(\text { Riemann })
$$

between the (linearized) Riemann tensor and the Beltrami parametrization.

Taking into account the factor 2 involved by multiplying the second, third and fifth row by 2 , we get the new $6 \times 6$ operator matrix with rank 3 :

$$
\left(\begin{array}{cccccc}
0 & 0 & 0 & d_{33} & -2 d_{23} & d_{22} \\
0 & -2 d_{33} & 2 d_{23} & 0 & 2 d_{13} & -2 d_{12} \\
0 & 2 d_{23} & -2 d_{22} & -2 d_{13} & 2 d_{12} & 0 \\
d_{33} & 0 & -2 d_{13} & 0 & 0 & d_{11} \\
-2 d_{23} & 2 d_{13} & 2 d_{12} & 0 & -2 d_{11} & 0 \\
d_{22} & -2 d_{12} & 0 & d_{11} & 0 & 0
\end{array}\right)
$$

clearly providing a self-adjoint operator.

Surprisingly, the Maxwell parametrization is obtained by keeping $\phi_{11}=A, \phi_{22}=B, \phi_{33}=C$ while setting $\phi_{12}=\phi_{23}=\phi_{31}=0$ in order to obtain the system:

$$
\left\{\begin{array}{l|lll}
\sigma^{11} \equiv d_{33} B+d_{22} C=0 & 1 & 2 & 3 \\
\sigma^{22} \equiv d_{33} A+d_{11} C=0 & 1 & 2 & 3 \\
-\sigma^{23} \equiv d_{23} A=0 & 1 & 2 & \bullet \\
\sigma^{33} \equiv d_{22} A+d_{11} B=0 & 1 & 2 & \bullet \\
-\sigma^{13} \equiv d_{13} B=0 & 1 & \bullet & \bullet \\
-\sigma^{12} \equiv d_{12} C=0 & 1 & \bullet
\end{array}\right.
$$


However, this system may not be involutive and no CC can be found "a priori" because the coordinate system is surely not $\delta$-regular. Indeed, effecting the linear change of coordinates $\bar{x}^{1}=x^{1}, \bar{x}^{2}=x^{2}, \bar{x}^{3}=x^{3}+x^{2}+x^{1}$ and taking out the bar for simplicity, we obtain the new involutive system:

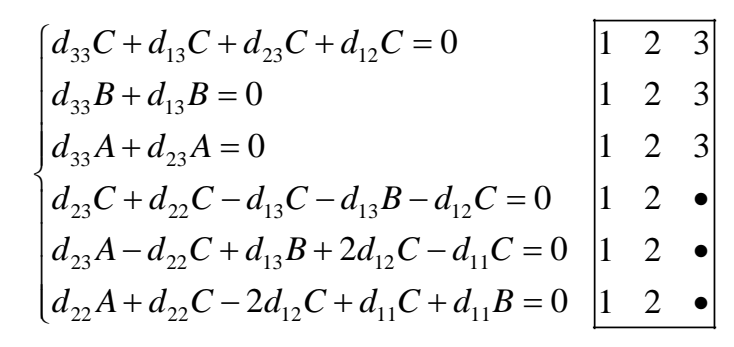

and it is easy to check that the $3 \mathrm{CC}$ obtained just amount to the desired 3 stress equations when coming back to the original system of coordinates. However, the three characters are different as we have now

$\alpha_{2}^{3}=3-3=0<\alpha_{2}^{2}=2 \times 3-3=3<\alpha_{2}^{1}=3 \times 3-0=9$ with sum equal to $\operatorname{dim}\left(g_{2}\right)=6 \times 3-6=18-6=12$. We have thus a minimum parametrization that cannot be parametrized again.

Again, if there is a geometrical background, this change of local coordinates is hidding it totally. Moreover, we notice that the stress functions kept in the procedure are just the ones on which $d_{33}$ is acting. The reason for such an apparently technical choice is related to very general deep arguments in the theory of differential modules that will only be explained at the end of the paper.

The Morera parametrization is obtained similarly by keeping now $\phi_{23}=L, \phi_{13}=M, \phi_{12}=N$ while setting $\phi_{11}=\phi_{22}=\phi_{33}=0$, namely:

$$
\left\{\begin{array}{l}
d_{23} L=0 \\
d_{33} N-d_{13} L-d_{23} M=0 \\
d_{13} M=0 \\
d_{22} M-d_{23} N-d_{12} L=0 \\
d_{11} L-d_{12} M-d_{13} N=0 \\
d_{12} N=0
\end{array}\right.
$$

Using now the same change of coordinates as the one already done for the Maxwell parametrization, we obtain the following system with 3 equations of (full) class 3 and 3 equations of class 2 in the Pommaret basis corresponding to the Janet tabular:

$$
\left\{\begin{array}{l}
d_{33} N+d_{23} N+d_{13} N+d_{12} N=0 \\
d_{33} M+d_{13} M=0 \\
d_{33} L+d_{23} L=0 \\
\left(d_{23} N+d_{23} M-d_{23} L\right)+\left(d_{13} N-d_{13} M+d_{13} L\right)+d_{12} N=0 \\
2 d_{23} M+\left(d_{13} N-d_{13} M-d_{13} L\right)+d_{12} M-d_{11} L=0 \\
d_{22} M+\left(d_{12} N-d_{12} M-d_{12} L\right)+d_{11} L=0
\end{array} \mid \begin{array}{lll}
1 & 2 & 3 \\
1 & 2 & 3 \\
1 & 2 & 3 \\
1 & 2 & \bullet \\
1 & 2 & \bullet \\
1 & 2 & \bullet
\end{array}\right.
$$

After elementary but tedious computations (that could not be avoided!), one can prove that the $3 \mathrm{CC}$ corresponding to the 3 dots are effectively satisfied and 
that they correspond to the 3 Cauchy stress equations which are therefore parametrized. The parametrization is thus provided by an involutive operator defining a torsion module because the character $\alpha_{2}^{3}$ is vanishing in $\delta$-regular coordinates, just like before for the Maxwell parametrization. We have thus another minimum parametrization that cannot be parametrized again. Of course, such a result could not have been understood by Beltrami in 1892 because the work of Cartan could not be adapted easily as it is using the language of exterior forms and the work of Janet only appeared in 1920 with no explicit reference to involution because only Janet bases are used [8] while the Pommaret bases have only been introduced in 1978 [1].

On a purely computational level, we may also keep only $\left\{\phi_{11}, \phi_{12}, \phi_{22}\right\}$ and obtain the different involutive system with the same characters and, in particular, $\alpha_{2}^{3}=0$ :

$$
\left\{\begin{array}{l|l}
\sigma^{11} \equiv d_{33} \phi_{22}=0 \\
-\sigma^{12} \equiv d_{33} \phi_{12}=0 \\
\sigma^{22} \equiv d_{33} \phi_{11}=0 \\
\sigma^{13} \equiv d_{23} \phi_{12}-d_{13} \phi_{22}=0 \\
-\sigma^{23} \equiv d_{23} \phi_{11}-d_{13} \phi_{12}=0 \\
\sigma^{33} \equiv d_{22} \phi_{11}+d_{11} \phi_{22}-2 d_{12} \phi_{12}=0
\end{array}\right) \begin{array}{lll}
1 & 2 & 3 \\
1 & 2 & 3 \\
1 & 2 & 3 \\
1 & 2 & \bullet \\
1 & 2 & \bullet \\
1 & 2 & \bullet
\end{array}
$$

So far, we have thus obtained three explicit local minimum parametrizations of the Cauchy stress equations with $n(n-1) / 2=3$ stress potentials but there may be others [21].

- $n=4$ : It just remains to explain the relation of the previous results with Einstein equations. The first surprising link is provided by the following technical proposition:

PROPOSITION 3.1: The Beltrami parametrization is just described by the Einstein operator when $n=3$. The same confusion existing between the Bianchi operator and the Cauchy operator has been made by both Einstein and Beltrami because the Einstein operator and the Beltrami operator are self-adjoint in arbitrary dimension $n \geq 3$, contrary to the Ricci operator.

Proof: The number of components of the Riemann tensor is $\operatorname{dim}\left(F_{1}\right)=n^{2}\left(n^{2}-1\right) / 12$. We have the combinatorial formula $n^{2}\left(n^{2}-1\right) / 12-n(n+1) / 2=n(n+1)(n+2)(n-3) / 12$ expressing that the number of components of the Riemann tensor is always greater or equal to the number of components of the Ricci tensor whenever $n>2$. Also, we have shown in many books [1] [2] [3] [4] [15] [19] or papers [10] [21] [29] [30] that the number of Bianchi identities is equal to $n^{2}\left(n^{2}-1\right)(n-2) / 24$, that is 3 when $n=3$ and 20 when $n=4$. Of course, it is well known that the divoperator, induced as CC of the Einstein operator, has $n$ components in arbitrary dimension $n \geq 3$.

Accordingly, when $n=3$ we have $n^{2}\left(n^{2}-1\right) / 12=n(n+1) / 2=6$ and it only remains to prove that the Einstein operator reduces to the Beltrami operator and not just to the Ricci operator. The following formulas can be found in any textbook on general relativity: 
Hence the difference can only be seen when $\omega_{i \neq j}=0$. In our situation with $n=3$ and the Euclidean metric for simplicity, we have:

$$
\begin{aligned}
2 R_{12}=2 E_{12}= & \left(d_{11}+d_{22}+d_{33}\right) \Omega_{12}+d_{12}\left(\Omega_{11}+\Omega_{22}+\Omega_{33}\right) \\
& -\left(d_{11} \Omega_{12}+d_{12} \Omega_{22}+d_{13} \Omega_{23}\right)-\left(d_{12} \Omega_{11}+d_{22} \Omega_{12}+d_{23} \Omega_{13}\right) \\
=d_{33} \Omega_{12} & +d_{12} \Omega_{33}-d_{13} \Omega_{23}-d_{23} \Omega_{13} \\
2 R_{11}= & \left(d_{11}+d_{22}+d_{33}\right) \Omega_{11}+d_{11}\left(\Omega_{11}+\Omega_{22}+\Omega_{33}\right) \\
& -2\left(d_{11} \Omega_{11}+d_{12} \Omega_{12}+d_{13} \Omega_{13}\right) \\
= & \left(d_{22}+d_{33}\right) \Omega_{11}+d_{11}\left(\Omega_{22}+\Omega_{33}\right)-2\left(d_{12} \Omega_{12}+d_{13} \Omega_{13}\right) \\
\operatorname{tr}(R)= & \left(d_{11} \Omega_{22}+d_{11} \Omega_{33}+d_{22} \Omega_{11}+d_{22} \Omega_{33}+d_{33} \Omega_{11}+d_{33} \Omega_{22}\right) \\
& -2\left(d_{12} \Omega_{12}+d_{13} \Omega_{13}+d_{23} \Omega_{23}\right) \\
& -2 E_{11}=d_{22} \Omega_{33}+d_{33} \Omega_{22}-2 d_{23} \Omega_{23}
\end{aligned}
$$

In the light of modern differential geometry, comparing these results with the works of both Maxwell, Morera, Beltrami and Einstein, it becomes clear that they have been confusing the div operator induced from the Bianchi operator with the Cauchy operator. However, it is also clear that they both obtained a possibility to parametrize the Cauchy operator by means of 3 arbitrary potential like functions in the case of Maxwell and Morera, 6 in the case of Beltrami who explains the previous choices, and 10 in the case of Einstein. Of course, as they were ignoring that the Einstein operator was self-adjoint whenever $n \geq 3$, they did not notice that we have Cauchy=ad(Killing) and they were unable to compare their results with the Airy operator found as early as in 1870 for the same mechanical purpose when $n=2$. To speak in a rough way, the situation is similar to what could happen in the study of contact structures if one should confuse $\mathcal{D}_{-1}$ with $\mathcal{D}_{1}$ [29]. Finally, using Theorem 2.1 or Proposition 2.2, we can choose a differential transcendence basis with $n(n-1) / 2$ potentials that can be indexed by $\phi_{i j}=\phi_{j i}$ with $i<j$ or $1 \leq i, j \leq n-1$ or even $2 \leq i, j \leq n$ when the dimension $n \geq 2$ is arbitrary (see [2] or [40] for more details on differential algebra).

Q.E.D.

REMARK 3.2: In the opinion of the author of this paper who is not a historian of sciences but a specialist of mathematical physics interested in the analogy existing between electromagnetism (EM), elasticity(EL) and gravitation (GR) by using the conformal group of space-time (see [3] [10] [28] [29] [30] [41] [42] [43] [44] for related works), it is difficult to imagine that Einstein could not have been aware of the works of Maxwell and Beltrami on the foundations of EL and tensor calculus. Indeed, not only they were quite famous when he started his research work but it must also be noticed that the Mach-Lippmann analogy [45] [46] [47] [48] was introduced at the same time (see [3] and [49] for more details on the field-matter couplings and the phenomenological law discovered by... Maxwell too). The main idea is that classical variational calculus using a Lagran- 
gian formalism must be only considered as the basic scheme of a more general and powerful "duality theory" that only depends on new purely mathematical tools, namely "group theory" and "differential homological algebra" (see [4] or [15] for the theory and [21] for the applications).

The two following crucial results, still neither known nor acknowledged today, are provided by the next proposition and corresponding corollary [40]:

PROPOSITION 3.3: The Cauchyoperator can be parametrized by the formal adjoint of the Ricci operator (4 terms) and the Einstein operator (6 terms) is thus useless. The so-called gravitational waves equations are thus nothing else than the formal adjoint of the linearized Ricci operator.

Proof: The Einstein operator $\Omega \rightarrow E$ is defined by setting $E_{i j}=R_{i j}-\frac{1}{2} \omega_{i j} t r(R)$ that we shall write Einstein $=C \circ$ Ricci where $C: S_{2} T^{*} \rightarrow S_{2} T^{*}$ is a symmetric matrix only depending on $\omega$, which is invertible whenever $n \geq 3$. Surprisingly, we may also introduce the same linear transformation $C: \Omega \rightarrow \bar{\Omega}=\Omega-\frac{1}{2} \omega \operatorname{tr}(\Omega)$ and the unknown composite operator $\mathcal{X}: \bar{\Omega} \rightarrow \Omega \rightarrow E$ in such a way that Einstein $=\mathcal{X} \circ C$ where $\mathcal{X}$ is defined by (see [33], 5.1.5 p 134):

$$
\begin{aligned}
2 E_{i j}= & \omega^{r s} d_{r s} \bar{\Omega}_{i j}-\omega^{r s} d_{r i} \bar{\Omega}_{s j}-\omega^{r s} d_{s j} \bar{\Omega}_{r i} \\
& +\omega_{i j} \omega^{r u} \omega^{s v} d_{r s} \bar{\Omega}_{u v}
\end{aligned}
$$

Now, introducing the test functions $\lambda^{i j}$, we get:

$$
\begin{aligned}
& \lambda^{i j} E_{i j}=\lambda^{i j}\left(R_{i j}-\frac{1}{2} \omega_{i j} \operatorname{tr}(R)\right) \\
& =\left(\lambda^{i j}-\frac{1}{2} \lambda^{r s} \omega_{r s} \omega^{i j}\right) R_{i j}=\bar{\lambda}^{i j} R_{i j}
\end{aligned}
$$

Integrating by parts while setting as usual $\square=\omega^{r s} d_{r s}$, we obtain:

$$
\left(\square \bar{\lambda}^{r s}+\omega^{r s} d_{i j} \bar{\lambda}^{i j}-\omega^{s j} d_{i j} \bar{\lambda}^{r i}-\omega^{r i} d_{i j} \bar{\lambda}^{s j}\right) \Omega_{r s}=\sigma^{r s} \Omega_{r s}
$$

Moreover, suppressing the "bar" for simplicity, we have:

$$
\begin{aligned}
d_{r} \sigma^{r s} & =\omega^{i j} d_{r i j} \lambda^{r s}+\omega^{r s} d_{r i j} \lambda^{i j}-\omega^{s j} d_{r i j} \lambda^{r i}-\omega^{r i} d_{r i j} \lambda^{s j} \\
& =0
\end{aligned}
$$

As Einstein is a self-adjoint operator (contrary to the Ricci operator), we have the identities:

$$
\begin{aligned}
& \operatorname{ad}(\text { Einstein })=\operatorname{ad}(C) \circ \operatorname{ad}(\mathcal{X}) \Rightarrow \text { Einstein }=C \circ \operatorname{ad}(\mathcal{X}) \\
& \Rightarrow \operatorname{ad}(\mathcal{X})=\operatorname{Ricci} \Rightarrow \mathcal{X}=\operatorname{ad}(\text { Ricci })
\end{aligned}
$$

Indeed, $a d(C)=C$ because $C$ is a symmetric matrix and we know that ad $($ Einstein $)=$ Einstein . Accordingly, the operator ad (Ricci) parametrizes the Cauchy equations, without any reference to the Einstein operator which has no mathematical origin, in the sense that it cannot be obtained by any diagram chasing. The three terms after the Dalembert operator factorize through the di- 
vergence operator $d_{i} \lambda^{r i}$. We may thus add the differential constraints $d_{i} \lambda^{r i}=0$ without any reference to a gauge transformation in order to obtain a (minimum) relative parametrization (see [14] and [18] for details and explicit examples). When $n=4$ we finally obtain the adjoint sequences:

$$
4 \underset{\quad \stackrel{\text { Killing }}{\rightarrow}}{\rightarrow} 10 \stackrel{\text { Ricci }}{\rightarrow} 10
$$

without any reference to the Bianchi operator and the induced div operator.

Finally, using Theorem 2.1 or Proposition 2.2, we may choose a differential transcendence basis made by $\left\{\lambda^{i j} \mid i<j\right\}$ or $\left\{\lambda^{i j} \mid 1<i, j<n-1\right\}$ or even $\left\{\lambda^{i j} \mid 2<i, j<n\right\}$ when the dimension $n \geq 2$ is arbitrary (see again [2] or [40] for more details on differential algebra).

Q.E.D.

COROLLARY 3.4: The differential module $N$ defined by the Ricci or the Einstein operator is not torsion-free and cannot therefore be parametrized. Its torsion submodule is generated by the 10 components of the Weyl operator that are separately killed by the Dalembert operator.

Proof: In order to avoid using extension modules, we present the 5 steps of the double differential duality test in this framework:

Step 1: Start with the Einstein operator $\mathcal{D}_{1}: 10 \stackrel{\text { Einstein }}{\rightarrow} 10$.

Step 2: Consider its formal adjoint: $\operatorname{ad}\left(\mathcal{D}_{1}\right): 10 \stackrel{\text { Einstein }}{\leftarrow} 10$.

Step 3: Compute the generating CC, namely the Cauchyoperator: $\operatorname{ad}(\mathcal{D}): 4 \stackrel{\text { Cauchy }}{\leftarrow} 10$.

Step 4: Consider its formal adjoint: $\mathcal{D}=\operatorname{ad}(\operatorname{ad}(\mathcal{D})): 4 \stackrel{\text { Killing }}{\rightarrow} 10$.

Step 5: Compute the generating CC, namely the Riemann operator: $\mathcal{D}_{1}^{\prime}: 10 \stackrel{\text { Riemann }}{\rightarrow} 20$.

With a slight abuse of language, we have the direct sum Riemann $=$ Ricci $\oplus$ Weyl with $20=10+10$. It follows from differential homological algebra that the 10 additional CC in $\mathcal{D}_{1}^{\prime}$ that are not in $\mathcal{D}_{1}$, are generating the torsion submodule $t(N)$ of the differential module $N$ defined by the Einstein or Ricci operator. In general, if $K$ is a differential field with commuting derivations $\partial_{1}, \cdots, \partial_{n}$, we way consider the ring $D=K\left[d_{1}, \cdots, d_{n}\right]=K[d]$ of differential operators with coefficients in $K$ and it is known that $r k_{D}(\mathcal{D})=r k_{D}(\operatorname{ad}(\mathcal{D}))$ for any operator matrix $\mathcal{D}$ with coefficients in $K$. In the present situation, as the Minkowski metric has coefficients equal to $0,1,-1$, we may choose the ground differential field to be $K=\mathbb{Q}$. Hence, there exist operators $\mathcal{P}$ and $\mathcal{Q}$ such that we have an identity:

$$
\mathcal{P} \circ \text { Weyl }=\mathcal{Q} \circ \text { Ricci }
$$

One may also notice that $r k_{D}($ Einstein $)=r k_{D}($ Ricci $)$ with: 


$$
\begin{aligned}
& r k_{D}(\text { Einstein })=\frac{n(n+1)}{2}-n=\frac{n(n-1)}{2}, \\
& r k_{D}(\text { Riemann })=\frac{n(n+1)}{2}-n=\frac{n(n-1)}{2}
\end{aligned}
$$

The differential ranks of the Einstein and Riemann operators are thus equal, but this is a pure coincidence because $r k_{D}$ (Einstein) has only to do with the divoperator induced by contracting the Bianchi operator, while $r k_{D}$ (Riemann) has only to do with the classical Killing operator and the fact that the corresponding differential module is a torsion module because we have a Lie group of transformations having $n+\frac{n(n-1)}{2}=\frac{n(n+1)}{2}$ parameters (translations + rotations). Hence, as the Riemann operator is a direct sum of the Weyloperator and the Einstein or Ricci operator according to the previous theorem, each component of the Weyl operator must be killed by a certain operator whenever the Einstein or Ricci equations in vacuum are satisfied. It is not at all evident that we have $\mathcal{P}=\square$ acting on each component of the Weyl operator. A direct tricky computation can be found in ([49], p 206), ([50], exercise 7.7) and ([15], p 95). With more details, we may start from the long exact sequence:

$$
0 \rightarrow \Theta \rightarrow 4 \stackrel{\text { Killing }}{\rightarrow} 10 \stackrel{\text { Riemann }}{\rightarrow} 20 \stackrel{\text { Bianchi }}{\rightarrow} 20 \rightarrow 6 \rightarrow 0
$$

This resolution of the set of Killing vector fields is not a Janet sequence because the Killing operator is not involutive as it is an operator of finite type with symbol of dimension $n(n-1) / 2=6$ and one should need one prolongation for getting an involutive operator with vanishing second order symbol. Splitting the Riemann operator we get the commutative and exact diagram:

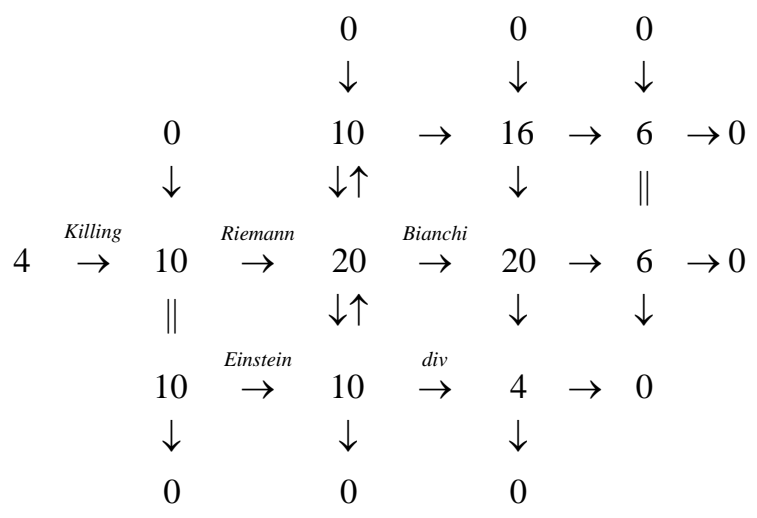

Passing to the module point of view, we have the long exact sequence:

$$
0 \rightarrow D^{6} \rightarrow D^{20} \stackrel{\text { Bianchi }}{\rightarrow} D^{20} \stackrel{\text { Riemann }}{\rightarrow} D^{10} \stackrel{\text { Killing }}{\rightarrow} D^{4} \rightarrow M \rightarrow 0
$$

which is a resolution of the Killing differential module $M=$ coker (Killing) and we check that we have indeed the vanishing of the Euler-Poincaré characteristic $6-20+20-10+4=0$. Accordingly, we have $N^{\prime}=\operatorname{coker}($ Riemann $) \simeq \operatorname{im}($ Killing $) \subset D^{4}$ and thus $N^{\prime}$ is torsion-free with 
$r k_{D}\left(N^{\prime}\right)=4-0=4=n$ because $r k_{D}(M)=0$.

We have the following commutative and exact diagram where $N=\operatorname{coker}($ Einstein $)$ :

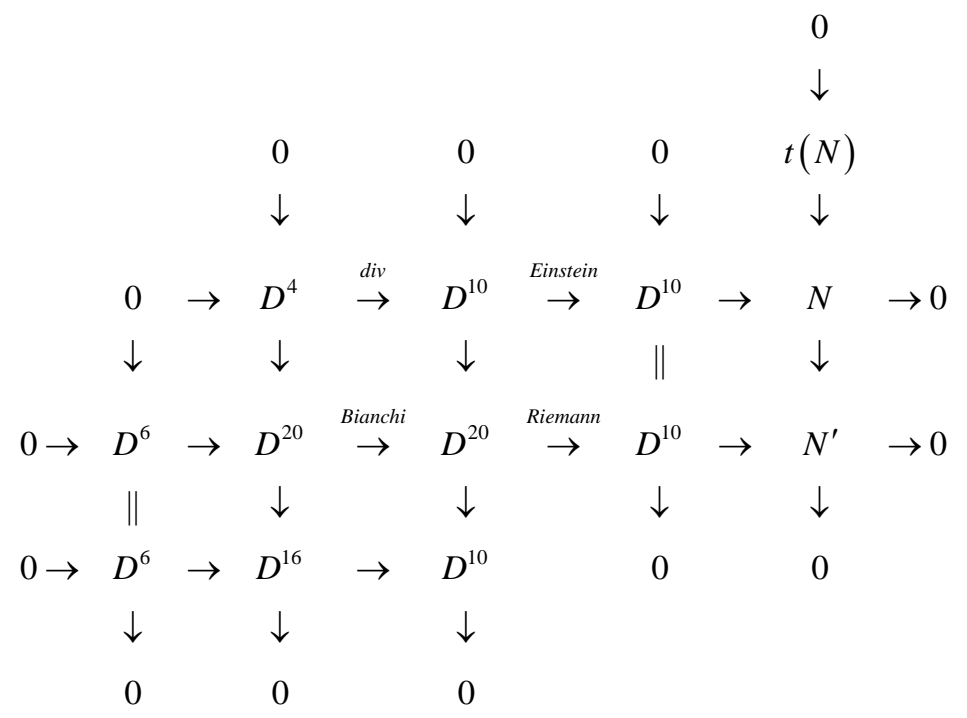

If $L$ is the kernel of the epimorphism $N \rightarrow N^{\prime}$, it is a torsion module because $r k_{D}(L)=r k_{D}(N)-r k_{D}\left(N^{\prime}\right)=4-4=0$. We have thus $L \subseteq t(N)$ in the following commutative and exact diagram:

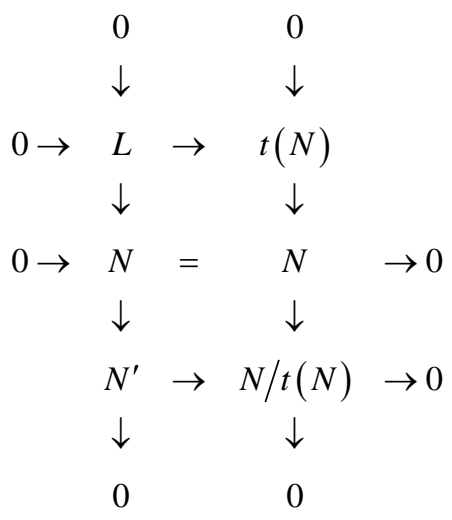

where $N / t(N)$ is a torsion-free module by definition. A snake chase allows to prove that the cokernel of the monomorphism $L \rightarrow t(N)$ is isomorphic to the kernel of the induced epimorphism $N^{\prime} \rightarrow N / t(N)$ and must be therefore, at the same time, a torsion module because $r k_{D}(L)=r k_{D}(t(N))=0$ and a torsion-free module because $N^{\prime} \subset D^{4}$, a result leading to a contradiction unless it is zero and thus $L=t(N)$. A snake chase in the previous diagram allows to exhibit the long exact connecting sequence:

$$
0 \rightarrow D^{6} \rightarrow D^{16} \rightarrow D^{10} \rightarrow t(N) \rightarrow 0
$$

It must be noticed that one cannot find canonical morphisms between the classical and conformal resolutions constructed similarly because we recall that, for $n=4$ (only), the CC of the Weyl operator are of order 2 and not 1 like the Bianchi CC for the Riemann operator (see [37] for a computer algebra check- 
ing !). However, it follows from the last theorem that the short exact sequence $0 \rightarrow D^{10} \rightarrow D^{20} \rightarrow D^{10} \rightarrow 0$ splits with $D^{20} \simeq D^{10} \oplus D^{10}$ but the existence of a canonical lift $D^{20} \rightarrow D^{10} \rightarrow 0$ in the above diagram does not allow to split the right column and thus $N \neq N^{\prime} \oplus t(N)$ as $N^{\prime}$ is not even free. Hence, one can only say that the space of solutions of Einstein equations in vacuum contains the generic solutions of the Riemann operator which are parametrized by arbitrary vector fields. As for the torsion elements, we have $t(N)=\operatorname{coker}\left(D^{16} \rightarrow D^{10}\right)$ and we may thus represent them by the components of the Weyl tensor, killed by the Dalembertian. This module interpretation may thus question the proper origin and existence of gravitational waves because the div operator on the upper left part of the diagram has strictly nothing to do with the Cauchy = ad (Killing) operator which cannot appear anywhere in this diagram.

Q.E.D.

COROLLARY 3.5: More generally, when $\mathcal{D}$ is a Lie operator of finite type, that is when $[\Theta, \Theta] \subset \Theta$ under the ordinary bracket of vector fields or $\left[R_{q}, R_{q}\right] \subset R_{q}$ under the bracket of Lie algebroids and $g_{q+r}=0$ for $r$ large enough, then the Spencer sequence is locally isomorphic to the tensor product of the Poincaré sequence for the exterior derivative by a finite dimensional Lie algebra. It is thus formally exact both with its adjoint sequence. As it is known that the extension modules do not depend on the resolution used, this is the reason for which not only the Cauchyoperator can be parametrized but also the Cosserat couple-stress equations ad $\left(\mathcal{D}_{1}\right)$ can be parametrized by $\operatorname{ad}\left(\mathcal{D}_{2}\right)$, a result not evident at all (see [41] and [43] for explicit computations).

REMARK 3.6: A similar situation is well known for the Cauchy-Riemann equations when $n=2$. Indeed, any infinitesimal complex transformation $\xi$ must be solution of the linear first order homogeneous system $\xi_{2}^{2}-\xi_{1}^{1}=0, \xi_{2}^{1}+\xi_{1}^{2}=0$ of infinitesimal Lie equations though we obtain $\xi_{11}^{1}+\xi_{22}^{1}=0, \xi_{11}^{2}+\xi_{22}^{2}=0$, that is $\xi^{1}$ and $\xi^{2}$ are separately killed by the second order Laplace operator $\Delta=d_{11}+d_{22}$.

REMARK 3.7: A similar situation is also well known for the wave equations for the EM field $F$ in electromagnetism. Indeed, starting with the first set of Maxwell equations $d F=0$ and using the Minkowski constitutive law in vacuum with electric constant $\varepsilon_{0}$ and magnetic constant $\mu_{0}$ such that $\varepsilon_{0} \mu_{0} c^{2}=1$ for the seconf set of Maxwell equations, a standard tricky differential elimination allows to avoid the Lorenz (no " $\mathrm{t}$ ") gauge condition for the EM potential and to obtain directly $\square F=0$ (see [15] and [40] for the details).

Using computer algebra or a direct checking with the ordering $11<12<13<22<23<33$, we obtain:

$$
\begin{gathered}
E_{33}=\omega^{44} d_{44} \Omega_{33}+\text { lower terms } \\
E_{23}=\omega^{44} d_{44} \Omega_{23} \cdots
\end{gathered}
$$

We have therefore the following Janet tabular: 


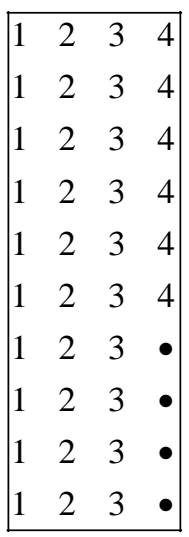

we are in the position to compute the characters of the Einstein operator but a similar procedure could be followed with the Ricci operator. We obtain at once:

$$
\begin{aligned}
& \beta_{2}^{4}=6 \Rightarrow \alpha_{2}^{4}=(10 \times 1)-6=4 \\
& \beta_{2}^{3}=4 \Rightarrow \alpha_{2}^{3}=(10 \times 2)-4=16 \\
& \beta_{2}^{2}=0 \Rightarrow \alpha_{2}^{2}=(10 \times 3)-0=30 \\
& \beta_{2}^{1}=0 \Rightarrow \alpha_{2}^{1}=(10 \times 4)-0=40
\end{aligned}
$$

a result leading to $\operatorname{dim}\left(g_{2}\right)=\alpha_{2}^{1}+\alpha_{2}^{2}+\alpha_{2}^{3}+\alpha_{2}^{4}=90$ and $\operatorname{dim}\left(g_{3}\right)=\alpha_{2}^{1}+2 \alpha_{2}^{2}+3 \alpha_{2}^{3}+4 \alpha_{2}^{4}=164$ along with the long exact sequences:

$$
\begin{aligned}
0 & \rightarrow g_{2} \rightarrow S_{2} T^{*} \otimes S_{2} T^{*} \rightarrow S_{2} T^{*} \rightarrow 0 \\
0 \rightarrow g_{3} & \rightarrow S_{3} T^{*} \otimes S_{2} T^{*} \rightarrow T^{*} \otimes S_{2} T^{*} \rightarrow T^{*} \rightarrow 0
\end{aligned}
$$

Now, we have by definition $\operatorname{div}=\left(d_{1}, d_{2}, d_{3}, d_{4}\right)$ and div $\circ$ Einstein $=(0,0,0,0)$.

As the Einstein operator is a self-adjoint $10 \times 10$ operator matrix up to a change of basis [27], we obtain therefore, with a slight abuse of language, $\operatorname{det}($ Einstein $)=0$ because:

$$
\left(\begin{array}{cccccccccc}
d_{1} & d_{2} & d_{3} & d_{4} & 0 & 0 & 0 & 0 & 0 & 0 \\
0 & d_{1} & 0 & 0 & d_{2} & d_{3} & d_{4} & 0 & 0 & 0 \\
0 & 0 & d_{1} & 0 & 0 & 0 & 0 & d_{2} & d_{3} & d_{4} \\
0 & 0 & 0 & d_{1} & 0 & 0 & d_{2} & 0 & d_{3} & d_{4}
\end{array}\right)\left(\begin{array}{l}
E_{11} \\
E_{12} \\
E_{13} \\
E_{14} \\
E_{22} \\
E_{23} \\
E_{24} \\
E_{33} \\
E_{34} \\
E_{44}
\end{array}\right)=\left(\begin{array}{lll}
0 & \cdots & 0 \\
0 & \cdots & 0 \\
0 & \cdots & 0 \\
0 & \cdots & 0
\end{array}\right)
$$

a result not evident at first sight that must be compared with the Poincaré situation when $n=3$ :

$$
\left(\begin{array}{lll}
d_{1} & d_{2} & d_{3}
\end{array}\right)\left(\begin{array}{ccc}
0 & -d_{3} & d_{2} \\
d_{3} & 0 & -d_{1} \\
-d_{2} & d_{1} & 0
\end{array}\right)=\left(\begin{array}{lll}
0 & 0 & 0
\end{array}\right)
$$




\section{Symbol Sequences}

A way to study the formal properties of a linear differential operator $\mathcal{D}: E \rightarrow F=F_{0}$ of order $q$ between the sections of two vector bundles over a manifold $X$ with dimension $n$ is to consider the symbol of $\mathcal{D}$ at the covector $\chi \in T^{*}(X)$, namely the map $\sigma_{\chi}(\mathcal{D})$ obtained from the leading terms of order $q$. With more details, if $\mathcal{D}$ is described by the operator matrix $\left(a_{k}^{\tau \mu} d_{\mu}\right)$ with $0 \leq|\mu| \leq q$ and $\chi=\chi_{i} d x^{i} \in T^{*}$, then $\sigma_{\chi}(\mathcal{D}): E \rightarrow F_{0}$ is described by the polynomial matrix $\left(a_{k}^{\tau \mu} \chi_{\mu}\right)$ with $|\mu|=q$. When $\mathcal{D}$ is formally integrable, then $\chi$ is said to be characteristic if $\sigma_{\chi}(\mathcal{D})$ fails to be injective and we may introduce the characteristic set $V$ as usual. When $\mathcal{D}$ is involutive, it is known after the work of M. Janet (1920) that one can construct first order operators $\mathcal{D}_{1}, \cdots, \mathcal{D}_{n}$, each one generating the compatibility conditions (CC) of the preceding one. However, the symbol maps $\sigma_{\chi}\left(\mathcal{D}_{i}\right): F_{i-1} \rightarrow F_{i}$ for $i=1, \cdots, n$ may not provide an exact sequence and any non-zero covector is characteristic for these operators. A comparison of the operators involved in a control system with $n=1$, in the study of the contact transformations with $n=3$ and of the linearized Einstein equations with $n=4$ proves that the preceding definition must be conveniently refined by saying that a covector is systatic if the matrix $\sigma_{\chi}(\mathcal{D})$ fails to have its maximum generic rank and the previous symbol sequence is exact otherwise and we may introduce the systatic set $W$ as in [1] [4] [5]. What we have done with the Janet sequence can also be done with the Spencer sequence that only involves first order operators induced by the Spencer operator. A close link with differential homological algebra and the corresponding differential extension modules can also be established and illustrated [14]. These new results are adding doubts to the ones we have already expressed on the origin and existence of gravitational waves as follows.

First of all, we must distinguish two cases:

- $\max _{\chi} r k\left(\sigma_{\chi}(\mathcal{D})\right)<m \Leftrightarrow \alpha_{q}^{n}>0$ : Any covector is characteristic and we have $W \subset V=T^{*}$ with a slight abuse of language. This is the situation for each of the operators $\mathcal{D}_{1}, \cdots, \mathcal{D}_{n}$.

- $\max _{\chi} r k\left(\sigma_{c h i}(\mathcal{D})\right)=m \Leftrightarrow \alpha_{q}^{n}=0$ : The symbol map $\sigma_{\chi}(\mathcal{D})$ fails to be injective if and only if all the $m \times m$ submatrices have vanishing determinants and we have $W=V \subset T^{*}$.

PROPOSITION 4.1: If $\mathcal{D}$ is involutive, the symbol sequence of the Janet sequence:

$$
0 \rightarrow \operatorname{ker}\left(\sigma_{\chi}(\mathcal{D})\right) \rightarrow E \stackrel{\sigma_{\chi}(\mathcal{D})}{\rightarrow} F_{0} \stackrel{\sigma_{\chi}\left(\mathcal{D}_{1}\right)}{\rightarrow} \cdots \stackrel{\sigma_{\chi}\left(\mathcal{D}_{n}\right)}{\rightarrow} F_{n} \rightarrow 0
$$

is exact if and only if $\chi \notin W$.

Proof. Using the Janet tabulars for $\mathcal{D}$ and $\mathcal{D}_{1}$ with $\operatorname{dim}(E)=m$, we get:

$$
\begin{aligned}
\operatorname{dim}\left(\operatorname{im}\left(\sigma_{\chi}(\mathcal{D})\right)\right) & =r k\left(\sigma_{\chi}(\mathcal{D})\right)=m-\alpha_{q}^{n}(\mathcal{D})=\beta_{q}^{n}(\mathcal{D}) \\
& =\alpha_{1}^{n}\left(\mathcal{D}_{1}\right)=\operatorname{dim}\left(\operatorname{ker}\left(\sigma_{\chi}\left(\mathcal{D}_{1}\right)\right)\right)
\end{aligned}
$$

and so on. 
When $n=3$, coming back to the Beltrami operator, that is the Einstein operator which is known to be involutive, we may change the local coordinates as we did for the Maxwell and Morera parametrizations. With $\bar{\chi}=d \bar{x}^{3}=d x^{1}+d x^{2}+d x^{3}=(1,1,1)$, then $\operatorname{ker}\left(\sigma_{\bar{\chi}}(\mathcal{D})\right)$ is defined by:

$$
\left\{\begin{array}{l}
\phi_{22}+\phi_{33}-2 \phi_{23}=0 \\
\phi_{12}+\phi_{33}-\phi_{23}-\phi_{13}=0 \\
\phi_{11}+\phi_{33}-2 \phi_{13}=0 \\
\phi_{12}+\phi_{23}-\phi_{13}-\phi_{22}=0 \\
\phi_{11}+\phi_{23}-\phi_{13}-\phi_{12}=0 \\
\phi_{11}+\phi_{22}-2 \phi_{12}=0
\end{array}\right.
$$

Multiplying the fourth equation by 2 and substituting the first, the third and the sixth, we get:

$$
\left(\phi_{11}+\phi_{22}\right)+\left(\phi_{22}+\phi_{33}\right)-\left(\phi_{11}+\phi_{33}\right)-2 \phi_{22}=0
$$

whenever $2 \phi_{23}=\phi_{22}+\phi_{33}, 2 \phi_{13}=\phi_{11}+\phi_{33}, 2 \phi_{12}=\phi_{11}+\phi_{22}$. This unexpected result, which has only to do with the use of $\delta$-regular local coordinates, is proving that the choice of the stress potentials has strictly nothing to do with the complementary cancellations respectively adopted by Maxwell or Morera according to Beltrami because, in both cases, $\bar{\chi} \notin V=W$ but $\chi=d x^{3}=(0,0,1) \in V=W$. This result can be extended to an arbitrary dimension.

\section{Conclusion}

After teaching elasticity for 25 years to high level students in some of the best french civil engineering schools, the author of this paper still keeps in mind one of the most fascinating exercises that he has set up. The purpose was to explain why a dam made with concrete is always vertical on the water-side with a slope of about 42 degrees on the other free side in order to obtain a minimum cost and the auto-stability under cracking of the surface under water (see the introduction of [5] for more details). Surprisingly, the main tool involved is the approximate computation of the Airy function inside the dam. The author discovered at that time that no one of the other teachers did know that the Airy parametrization is nothing else than the adjoint of the linearized Riemann operator used as generating CC for the deformation tensor by any engineer. Being involved in General Relativity (GR) at that time, it took him 25 years (1970-1995) to prove that the Einstein equations could not be parametrized [25] [26] [27]. However, nobody is a prophet in his own country and it is only recently that he discovered that GR could be considered as a way to parametrize the Cauchy operator. It follows that exactly the same confusion has been done by Maxwell, Morera, Beltrami and Einstein because, in all these cases, the operator considered is self-adjoint. As a byproduct, the variational formalism cannot allow to discover it as no engineer could have had in mind to confuse the deformation tensor with its CC in the 
Lagrangian used for finite elements computations. It is thus an open historical problem to know whether Einstein knew any one of the previous works done as all these researchers were quite famous at the time he was active. In our opinion at least, the comparison of the various parametrizations described in this paper needs no comment as we have only presented facts, just facts.

\section{Conflicts of Interest}

The author declares no conflicts of interest regarding the publication of this paper.

\section{References}

[1] Pommaret, J.-F. (1978) Systems of Partial Differential Equations and Lie Pseudogroups. Gordon and Breach, New York; Russian Translation: MIR, Moscow (1983).

[2] Pommaret, J.-F. (1983) Differential Galois Theory. Gordon and Breach, New York.

[3] Pommaret, J.-F. (1988) Lie Pseudogroups and Mechanics. Gordon and Breach, New York.

[4] Pommaret, J.-F. (1994) Partial Differential Equations and Group Theory. Kluwer, Dordrecht. https://doi.org/10.1007/978-94-017-2539-2

[5] Pommaret, J.-F. (2001) Partial Differential Control Theory. Kluwer, Dordrecht. https://doi.org/10.1007/978-94-010-0854-9

[6] Pommaret, J.-F. (2019) Journal of Modern Physics, 10, 371-401. https://doi.org/10.4236/jmp.2019.103025

[7] Spencer, D.C. (1965) Bulletin of the AMS, 75, 1-114.

[8] Janet, M. (1920) Journal de Mathematique, 8, 65-151.

[9] Pommaret, J.-F. (2005) Algebraic Analysis of Control Systems Defined by Partial Differential Equations. In: Advanced Topics in Control Systems Theory, Springer, Berlin, Lecture Notes in Control and Information Sciences 311, Chapter 5, 155-223. https://doi.org/10.1007/11334774 5

[10] Pommaret, J.-F. (2020) Journal of Modern Physics, 11, 1672-1710. https://doi.org/10.4236/jmp.2020.1110104

[11] Gasqui, J. (1982) Compositio Mathematica, 47, 43-69.

[12] Bjork, J.E. (1993) Analytic D-Modules and Applications. Kluwer, Dordrecht. https://doi.org/10.1007/978-94-017-0717-6

[13] Kashiwara, M. (1995) Algebraic Study of Systems of Partial Differential Equations. Mémoires de la Société Mathématique de France, 63 (Transl. from Japanese of His 1970 Master's Thesis).

[14] Pommaret, J.-F. (2015) Multidimensional Systems and Signal Processing, 26, 405-437. https://doi.org/10.1007/s11045-013-0265-0

[15] Pommaret, J.-F. (2018) New Mathematical Methods for Physics. Mathematical Physics Books, Nova Science Publishers, New York, 150 p.

[16] Schneiders, J.-P. (1994) Bulletin de la Société Royale des Sciences de Liège, 63, 223-295.

[17] Macaulay, F.S. (1916) The Algebraic Theory of Modular Systems. Cambridge Tract 19, Cambridge University Press, London. (Reprinted by Stechert-Hafner Service Agency, New York, 1964) https://doi.org/10.3792/chmm/1263317740 
[18] Pommaret, J.-F. (2012) Spencer Operator and Applications: From Continuum Mechanics to Mathematical Physics. In: Yong, G., Ed., Continuum Mechanics-Progress in Fundamentals and Engineering Applications, InTech, Rijeka, 1-32. https://doi.org/10.5772/35607

[19] Pommaret, J.-F. (2016) Deformation Theory of Algebraic and Geometric Structures. Lambert Academic Publisher (LAP), Saarbrucken.

http://arxiv.org/abs/1207.1964 https://doi.org/10.1007/BFb0083506

[20] Pommaret, J.-F. (2016) Journal of Modern Physics, 7, 699-728. https://arxiv.org/abs/1803.09610

[21] Pommaret, J.-F. (2019) Journal of Modern Physics, 10, 1454-1486. https://doi.org/10.4236/jmp.2019.1012097

[22] Hu, S.-T. (1968) Introduction to Homological Algebra. Holden-Day, San Francisco.

[23] Northcott, D.G. (1966) An Introduction to Homological Algebra. Cambridge University Press, Cambridge.

[24] Rotman, J.J. (1979) An Introduction to Homological Algebra (Pure and Applied Mathematics). Academic Press, Cambridge.

[25] Pommaret, J.-F. (1995) Comptes Rendus Académie des Sciences Paris, Série I, 320, 1225-1230.

[26] Zerz, E. (2000) Topics in Multidimensional Linear Systems Theory. Lecture Notes in Control and Information Sciences, LNCIS 256, Springer, Berlin.

[27] Pommaret, J.-F. (2013) Journal of Modern Physics, 4, 223-239. https://doi.org/10.4236/jmp.2013.48A022

[28] Pommaret, J.-F. (2014) Journal of Modern Physics, 5, 157-170. https://doi.org/10.4236/jmp.2014.55026

[29] Pommaret, J.-F. (2020) The Conformal Group Revisited. https://arxiv.org/abs/2006.03449

[30] Pommaret, J.-F. (2020) Nonlinear Conformal Electromagnetism and Gravitation. https://arxiv.org/abs/2007.01710

[31] Pommaret, J.-F. (2016) Journal of Modern Physics, 7, 699-728. https://doi.org/10.4236/jmp.2016.77068

[32] Pommaret, J.-F. and Quadrat, A. (1999) Systems \& Control Letters, 37, 247-260. https://doi.org/10.1016/S0167-6911(99)00030-4

[33] Foster, J. and Nightingale, J.D. (1979) A Short Course in General Relativity. Longman, London.

[34] Lanczos, C. (1962) Reviews of Modern Physics, 34, 379-389. https://doi.org/10.1103/RevModPhys.34.379

[35] Airy, G.B. (1863) Philosophical Transactions of the Royal Society of London, 153, 49-80. https://doi.org/10.1098/rstl.1863.0004

[36] Beltrami, E. (1892) Atti della Accademia Nazionale dei Lincei, 1, 141-142.

[37] Landriani, G.S. (2017) Meccanica, 52, 2801-2806. https://doi.org/10.1007/s11012-016-0611-z

[38] Maxwell, J.C. (1870) Transactions of the Royal Society of Edinburgh, 26, 1-40. https://doi.org/10.1017/S0080456800026351

[39] Morera, G. (1892) Atti della Accademia Nazionale dei Lincei, 1, 137-141 + 233-234.

[40] Pommaret, J.-F. (2017) Journal of Modern Physics, 8, 2122-2158. 
https://doi.org/10.4236/jmp.2017.813130

[41] Cosserat, E. and Cosserat, F. (1909) Théorie des Corps Déformables. Hermann, Paris.

[42] Pommaret, J.-F. (1997) Annales des Ponts et Chaussées, 82, 59-66.

[43] Pommaret, J.-F. (2010) Acta Mechanica, 215, 43-55. https://doi.org/10.1007/s00707-010-0292-y

[44] Pommaret, J.-F. (2019) Journal of Modern Physics, 10, 1566-1595. http://arxiv.org/abs/1802.02430 https://doi.org/10.4236/jmp.2019.1013104

[45] Adler, F.W. (1907) Annalen der Physik und Chemie, 22, 578-594. https://doi.org/10.1002/andp.19073270314

[46] Lippmann, G. (1876) Comptes rendus de P Académie des Sciences, 82, 1425-1428.

[47] Lippmann, G. (1907) Annalen der Physik und Chemie, 23, 994-996. https://doi.org/10.1002/andp.19073281017

[48] Mach, E. (1900) Prinzipien der Wärmelehre, 2, Aufl. J.A. Barth, Leipzig, 330.

[49] Choquet-Bruhat, Y. (2015) Introduction to General Relativity, Black Holes and Cosmology. Oxford University Press, Oxford.

[50] Hughston, L.P. and Tod, K.P. (1990) An Introduction to General Relativity. London Mathematical Society Student Texts 5. Cambridge University Press, Cambridge. https://doi.org/10.1017/CBO9781139171977 